\title{
November 21, 2018 \\ MONTE CARLO EULER APPROXIMATIONS OF HJM TERM STRUCTURE FINANCIAL MODELS
}

\author{
T. BJÖRK ${ }^{+}$, A. SZEPESSY ${ }^{\dagger}$, R. TEMPONE TEND G. E. ZOURARIS $^{\ddagger}$
}

\begin{abstract}
We present Monte Carlo-Euler methods for a weak approximation problem related to the Heath-Jarrow-Morton (HJM) term structure model, based on Itô stochastic differential equations in infinite dimensional spaces, and prove strong and weak error convergence estimates. The weak error estimates are based on stochastic flows and discrete dual backward problems, and they can be used to identify different error contributions arising from time and maturity discretization as well as the classical statistical error due to finite sampling. Explicit formulas for efficient computation of sharp error approximation are included. Due to the structure of the HJM models considered here, the computational effort devoted to the error estimates is low compared to the work to compute Monte Carlo solutions to the HJM model. Numerical examples with known exact solution are included in order to show the behavior of the estimates.
\end{abstract}

\section{THE HJM ModeL}

1.1. Generals. When valuing derivatives in the bond market it is important to use models that are consistent with the initial term structure observed in the market. The Heath-Jarrow-Morton (HJM) model for the forward rate has this property and in addition offers the freedom to choose the volatility structure, for example to be able to fit other derivative prices quoted in the market (see [6, 7, 15, 19]). This HJM model approach is particularly suitable for Monte Carlo computations, since in general the alternative of tree methods leads, for the multifactor case, to non recombining trees with higher computational cost.

In this work we focus on the numerical approximation of the price of financial instruments in the bond market, using the HJM model of forward rates. We propose Monte Carlo Euler methods fow which we develop a rigorous strong error analysis and provide rigorous weak error expansions, with leading error term in computable a posteriori form, offering computational reliability in the use of more complicated HJM multifactor models, where no explicit formula can be found, or such a formula is just too complicated to use, for the pricing of contingent claims. These weak error expansions can be used in adaptive algorithms to handle simultaneously different sources of error, e.g. time discretization, maturity discretization, and finite sampling, see [21]. To develop error estimates we use a Kolmogorov backward equation in an extended domain and carry out further the analysis in [21, from general weak approximation of Itô stochastic differential equations in $\mathbb{R}^{n}$, to weak approximation of the HJM Itô stochastic differential equations in infinite dimensional spaces. Therefore, the main new ingredient here is to provide error estimates useful for adaptive refinement not only in time $t$ but also in maturity time $\tau$. In addition, using the structure of the HJM model studied here, the application of a simple transformation removes the error caused by the representation of the initial term structure in a finite maturity partition. Finally, the formulas to compute sharp error approximations are simplified by exploiting the structure of the HJM model, reducing the work to compute such error estimates. The use of the error estimates proposed here is compatible with the application of variance reduction techniques, allowing for faster Monte Carlo computations, see 4 . 22 .

The work at hand is based on a research paper included in the one of the authors $\mathrm{PhD}$ Disseration

1991 Mathematics Subject Classification. Primary 65C05,65C30,65C20 Secondary 91B28,91B70 .

Key words and phrases. A priori error estimates, a posteriori error estimates, stochastic differential equations, Monte Carlo methods, HJM model, option price, bond market.

+ Institutionen för finansiell ekonomi, Handelshögskolan, Box 6501, S-113 83 Stockholm (Tomas.Bjork@hhs.se).

†Matematiska Institutionen, Kungl. Tekniska Högskolan, S-100 44 Stockholm (szepessy@kth.se).

$\S$ Division of Mathematics and Computational Sciences and Engineering (MCSE), 4700 King Abdullah University of Science and Technology (KAUST), Thuwal 23955-6900, Kingdom of Saudi Arabia (raul.tempone@kaust.edu.sa).

¥ Department of Mathematics, University of Crete, GR-714 09 Heraklion (zouraris@math.uoc.gr). 
1.2. Description of the model. The bond market is assumed to be efficient and without friction, i.e. there is no arbitrage opportunity, and there exists a martingale probability measure, under which bond contracts can be priced as expected values of properly discounted cash flows, see [1, 3, 9]. On what follows, all the equations are assumed to be under such a probability measure.

The HJM model is based on the so called forward rate, $f(t, \tau)$, which relates to the price of the most simple type of bond, the zero coupon bond, with contracting time $t$ and maturity time $\tau$, by

$$
p(t, \tau)=\exp \left(-\int_{t}^{\tau} f(t, \eta) d \eta\right) .
$$

In particular, the non arbitrage assumption in the HJM formulation, see 13, 14, yields an Itô stochastic differential equation, for $\tau \in\left[0, \tau_{\max }\right]$,

$$
\begin{aligned}
d f(t, \tau) & =\sum_{j=1}^{J} \sigma^{j}(t, \tau)\left(\int_{t}^{\tau} \sigma^{j}(t, s) d s\right) d t+\sum_{j=1}^{J} \sigma^{j}(t, \tau) d W^{j}(t), \quad t \in[0, \tau] \\
f(0, \tau) & =f_{0}(\tau) .
\end{aligned}
$$

Here $\left(W^{j}\right)_{j=1}^{J}$ are independent Wiener processes, and $\left(\sigma^{j}(t, \tau)\right)_{j=1}^{J}$ are stochastic processes, adapted to the filter structure generated by the Wiener processes. Furthermore, the initial datum for the term structure, $f_{0}:\left[0, \tau_{\max }\right] \rightarrow \mathbb{R}$, is a deterministic function in $C^{1}\left(\left[0, \tau_{\max }\right]\right)$. In this setting, the short rate, $r(t)$, is defined as $r(t) \equiv f(t, t)$.

On what follows the volatility function $\sigma=\left(\sigma^{1}, \ldots, \sigma^{J}\right)$ is assumed to be of the form

$$
\begin{aligned}
\sigma(t, \tau) & =\xi(r(t)) \lambda(t, \tau) \\
& =\xi(f(t, t)) \lambda(t, \tau),
\end{aligned}
$$

where $\xi: \mathbb{R} \rightarrow \mathbb{R}$ and $\lambda:\left[0, t_{\max }\right] \times\left[0, \tau_{\max }\right] \rightarrow \mathbb{R}^{J}$ are given bounded functions on $C^{m_{0}}(\mathbb{R})$ and $C^{m_{0}}\left(\left[0, t_{\max }\right] \times\left[0, \tau_{\max }\right]\right)$, respectively, for $m_{0}$ a sufficiently large integer. Then, setting

$$
\mathcal{D} \equiv\left\{(t, \tau) \in\left[0, t_{\max }\right] \times\left[0, \tau_{\max }\right]: t \leq \tau\right\}
$$

problem (1.1) reads as follows: find $f=f(t, \tau): \mathcal{D} \rightarrow \mathbb{R}$ such that

$$
\begin{aligned}
d f(t, \tau) & =\xi^{2}(f(t, t)) \widetilde{\lambda}(t, \tau) d t+\xi(f(t, t)) \lambda(t, \tau) \cdot d W(t), \quad t \in[0, \tau], \\
f(0, \tau) & =f_{0}(\tau)
\end{aligned}
$$

for $\tau \in\left[0, \tau_{\max }\right]$, where

$$
\widetilde{\lambda}(t, \tau) \equiv \lambda(t, \tau) \cdot \int_{t}^{\tau} \lambda(t, z) d z, \quad \forall t \in[0, \tau], \quad \forall \tau \in\left[0, \tau_{\max }\right] .
$$

Here the notation $a \cdot b$ denotes the standard inner product in $\mathbb{R}^{J}$, i.e. $a \cdot b \equiv \sum_{j=1}^{J} a_{j} b_{j}$. In many models used in practice, the function $\lambda$ has the form $\lambda(t, \tau)=\lambda_{0}(\tau-t)$, and then $\widetilde{\lambda}(t, \tau)=\widetilde{\lambda}_{0}(\tau-t)$ with

$$
\widetilde{\lambda}_{0}(\tau-t) \equiv \lambda_{0}(\tau-t) \cdot \int_{0}^{\tau-t} \lambda_{0}(z) d z .
$$

Observe that to solve for $f$ it is enough to have $\lambda_{0}: \mathbb{R}^{+} \rightarrow \mathbb{R}$. However, in this work the usual domain of definition $\mathcal{D}$ of $\lambda$ and $f$, extends to the set $\left[0, t_{\max }\right] \times\left[0, \tau_{\max }\right]$, leaving $\left.f\right|_{\mathcal{D}}$ unchanged. The extension of $\mathcal{D}$ helps to develop a posteriori approximations for the time and maturity discretization errors, depending on a linear backward problem (cf. Theorem 4.1).

A typical contract to price is a call option, with exercise time $t_{\max }$ and strike price $K$, on a zero coupon bond. Its price can be written in terms of the forward rate as

$$
\mathbb{E}\left[e^{-\int_{0}^{t_{\max }} f(s, s) d s} \max \left\{e^{-\int_{t_{\max }}^{\tau_{\max }} f\left(t_{\max }, \tau\right) d \tau}-K, 0\right\}\right] .
$$

Another basic contract is a continuous cap, with price

$$
\mathbb{E}\left[\int_{0}^{t_{\max }} e^{-\int_{0}^{t} f(s, s) d s}\left(f(t, t)-r_{c}\right)^{+} d t\right]
$$


where $r_{c}$ is a given value associated with the contract. With this motivation, and bearing in mind other possible contracts, we consider the approximation of the quantity

$$
\mathbb{E}[\mathcal{F}(f)]
$$

where the functional $\mathcal{F}(f)$ is given by

$$
\mathcal{F}(f) \equiv F\left(\int_{0}^{t_{\max }} f(s, s) d s\right) G\left(\int_{\tau_{a}}^{\tau_{\max }} \Psi\left(f\left(t_{\max }, \tau\right)\right) d \tau\right)+\int_{0}^{t_{\max }} F\left(\int_{0}^{s} f\left(s^{\prime}, s^{\prime}\right) d s^{\prime}\right) U(f(s, s)) d s
$$

with $\tau_{a}$ being a given positive number such that $0<t_{\max } \leq \tau_{a}<\tau_{\max }$. Obviously, $\mathcal{F}(f)$ is written equivalently as

$$
\mathcal{F}(f) \equiv F\left(Y\left(t_{\max }\right)\right) G\left(\Lambda\left(\Psi\left(f\left(t_{\max }, \cdot\right)\right)\right)\right)+Z\left(t_{\max }\right)
$$

where

$$
\begin{gathered}
Y(t) \equiv \int_{0}^{t} f(s, s) d s, \quad Z(t) \equiv \int_{0}^{t} F(Y(s)) U(f(s, s)) d s \\
\Lambda(w) \equiv \int_{\tau_{a}}^{\tau_{\max }} w(\tau) d \tau, \quad \forall w \in L^{1}\left(\tau_{a}, \tau_{\max }\right) .
\end{gathered}
$$

The functions $F: \mathbb{R} \rightarrow \mathbb{R}, G: \mathbb{R} \rightarrow \mathbb{R}, \Psi: \mathbb{R} \rightarrow \mathbb{R}, U: \mathbb{R} \rightarrow \mathbb{R}$, and their derivatives up to a sufficiently large order $m_{\star}$ are assumed to have a polynomial growth. We say that a function $S: \mathbb{R} \rightarrow \mathbb{R}$ has a polynomial growth if there exist positive constants $k^{\prime}$ and $C^{\prime}$ such that: $|S(x)| \leq C^{\prime}\left(1+|x|^{k^{\prime}}\right)$ for all $x \in \mathbb{R}$.

Let us consider the system of differential equations (1.2)-(1.3) describing the dynamics for the forward rate $f$ along with that for $Y(t)$ and $Z(t)$, i.e.,

$$
\begin{aligned}
d f(t, \tau) & =\xi^{2}(f(t, t)) \tilde{\lambda}(t, \tau) d t+\xi(f(t, t)) \lambda(t, \tau) \cdot d W(t), \\
d Y(t) & =f(t, t) d t \\
d Z(t) & =F(Y(t)) U(f(t, t)) d t
\end{aligned}
$$

for $t \in\left[0, t_{\max }\right]$ and $\tau \in\left[0 \tau_{\max }\right]$, with the initial conditions

$$
f(0, \tau)=f_{0}(\tau), \quad Y(0)=0, \quad Z(0)=0
$$

for $\tau \in\left[0 \tau_{\max }\right]$.

A approximation error for a typical discretization of the problem above will consists of a $t$-discretization error and a $\tau$-discretization error coming from the discretization of the initial condition $f_{0}$. Due to the special structure of (1.7)-(1.8), the initial error can be avoided and practically included in the $t$-discretization error by introducing the anzatz

$$
g(t, \tau)=f(t, \tau)-f_{0}(\tau),
$$

which implies $f(t, t)=g(t, t)+f_{0}(t)$. Thus, (1.7)-(1.8) is formulated as follows: find $g=g(t, \tau)$ : $\left[0, t_{\max }\right] \times\left[0, \tau_{\max }\right] \rightarrow \mathbb{R}$ such that

$$
\begin{aligned}
d g(t, \tau) & =\xi^{2}\left(g(t, t)+f_{0}(t)\right) \widetilde{\lambda}(t, \tau) d t+\xi\left(g(t, t)+f_{0}(t)\right) \lambda(t, \tau) \cdot d W(t), \quad \forall t \in\left[0, t_{\max }\right], \\
d Y(t) & =\left(g(t, t)+f_{0}(t)\right) d t \\
d Z(t) & =F(Y(t)) U\left(g(t, t)+f_{0}(t)\right) d t
\end{aligned}
$$

for $t \in\left[0, t_{\max }\right]$, with homogeneous initial conditions

$$
g(0, \tau)=0, \quad Y(0)=0, \quad Z(0)=0
$$

for all $\tau \in\left[0, \tau_{\max }\right]$. Thus, the quantity we want to approximate takes the form

$$
\mathbb{E}[\mathcal{G}(g)]
$$

where

$$
\mathcal{G}(g):=\mathcal{F}\left(g+f_{0}\right) .
$$


In the numerical methods, we describe later, the approximations to $Y$ and $Z$ will be always considered to be respectively the last two components of the approximate solution vector.

1.3. Overview. Let us give an overiview of the is organized as follows. In Section 2 first we present two Monte Carlo Euler methods for the HJM model (1.9)-(1.10), namely, a stochastic finite difference method, the Euler Finite Difference method (EFD), and a more accurate stochastic finite element method, the Euler Finite Element method (EFE); then, we combine a numerical quadrature rule and the outcome of the (EFD) or the (EFE) methods to construct a numerical approximation of the functional $\mathbb{E}[\mathcal{G}(g)]$. In Section 3 we provide a stong convergence analysis for the (EFD) and the (EFE) methods. Section 4 states and proves weak error estimates for the (EFD) method, giving explicit formulas for efficient computation of the discrete duals. Finally, Section 5 presents results from numerical experiments.

\section{Monte Carlo Euler Methods}

In this section first we introduce two time and maturity time discretizations of (1.9)-(1.10): the EulerFinite Difference (EFD) method and the Euler-Finite Element (EFE) method. Then, we use the (EFD) or the (EFE) approximations along with a quadrature rule to construct approximations of the quantity of interest $\mathbb{E}[\mathcal{G}(g)]$ defined in (1.11).

2.1. Time and maturity time discretization. Given extreme points $0<t_{\max } \leq \tau_{a}<\tau_{\max }$ introduced in Section 1 , let $N$ and $L$ denote the number of subintervals on $\left[0, t_{\max }\right]$ and $\left[0, \tau_{\max }\right]$, respectively. Then, consider partitions

$$
0=t_{0}<\cdots<t_{N}=t_{\max } \text { and } 0=\tau_{0}<\cdots<\tau_{L}=\tau_{\max }
$$

of the $t$-interval $\left[0, t_{\max }\right]$ and of the $\tau$-interval $\left[0, \tau_{\max }\right]$, respectively. For technical reasons, these partitions are assumed to satisfy the following condition: every $\tau$-node in the interval $\left[0, t_{\max }\right]$ is also a $t$-node, i.e.

$$
\text { there exists an one-to-one index map } \rho \text {, such that, } \tau_{\ell}=t_{\rho(\ell)} \text { for } \tau_{\ell} \leq t_{\max } \text {. }
$$

In addition, assume that

$$
\text { there exists an index } \ell_{\star} \text { such that } t_{\max }=\tau_{\ell_{\star}}
$$

and

$$
\text { there exists an index } \ell_{a} \text { such that } \tau_{a}=\tau_{\ell_{a}} \text {. }
$$

Also, define the auxiliary index function, $\ell_{n}$, by

$$
\ell_{n} \equiv \max \left\{\ell \in \mathbb{Z}: \quad 0 \leq \ell \leq L \quad \text { such that } \tau_{\ell} \leq t_{n}\right\}
$$

introduce the notation

$$
\begin{gathered}
\Delta t_{n} \equiv t_{n+1}-t_{n}, \quad \Delta W_{n} \equiv W\left(t_{n+1}\right)-W\left(t_{n}\right) \quad \text { for } \quad n=0, \ldots, N-1, \\
\Delta \tau_{\ell} \equiv \tau_{\ell+1}-\tau_{\ell} \quad \text { for } \quad \ell=0, \ldots, L-1,
\end{gathered}
$$

and set $\Delta t \equiv \max _{0 \leq n \leq N-1} \Delta t_{n}$ and $\Delta \tau \equiv \max _{0 \leq \ell \leq L-1} \Delta \tau_{\ell}$. Finally, introduce the space of piecewise constant and right continuous functions on a $\tau$-partition, $\left(\tau_{\ell}\right)_{\ell=0}^{L}$, of the interval $\left[0, \tau_{\max }\right]$, by

$$
S_{\Delta \tau} \equiv\left\{\chi \in L^{\infty}\left(0, \tau_{\max }\right): \text { there are constants }\left(c_{\ell}\right)_{\ell=0}^{L-1} \text { such that }\left.\chi\right|_{\left[\tau_{\ell}, \tau_{\ell+1}\right)}=c_{\ell}, \quad \ell=0, \ldots, L-1\right\} .
$$

Define the standard $L^{2}$-projection $\Pi: L^{2}\left(0, \tau_{\max }\right) \rightarrow S_{\Delta \tau}$ by

$$
\int_{0}^{\tau_{\max }} \Pi v \chi d \tau=\int_{0}^{\tau_{\max }} v \chi d \tau, \quad \forall \chi \in S_{\Delta \tau}, \quad \forall v \in L^{2}\left(0, \tau_{\max }\right),
$$

which satisfies

$$
\left.\Pi v\right|_{\left[\tau_{\ell}, \tau_{\ell+1}\right)}=\frac{1}{\Delta \tau_{\ell}} \int_{\tau_{\ell}}^{\tau_{\ell+1}} v(\tau) \quad d \tau, \quad \ell=0, \ldots, L-1, \quad \forall v \in L^{2}\left(0, \tau_{\max }\right) .
$$

For $\chi \in S_{\Delta \tau}$ and $\ell=0, \ldots, L-1$, denote by $\chi_{\ell}$ the constant value of $\chi$ in $\left[\tau_{\ell}, \tau_{\ell+1}\right)$. When considering a function, $w=w(t, \tau)$, depending on two variables, the $L^{2}$ projection is always with respect to $\tau$, i.e. for $\ell=0, \ldots, L-1$ and $\tau \in\left[\tau_{\ell}, \tau_{\ell+1}\right)$, we have $\left.\Pi w(t ; \tau) \equiv \Pi(w(t, \cdot))\right|_{\left[\tau_{\ell}, \tau_{\ell+1}\right)}=\frac{1}{\Delta \tau_{\ell}} \int_{\tau_{\ell}}^{\tau_{\ell+1}} w(t, s) d s$. 
2.2. The Euler-Finite Difference (EFD) method. For each time level the (EFD) method approximates $g\left(t_{n},.\right)$ by a piecewise constant function, $\overline{\bar{g}}\left(t_{n},.\right) \in S_{\Delta \tau}$. In particular, it finds the approximate values $\overline{\bar{g}}_{n, \ell} \approx g\left(t_{n}, \tau_{\ell}\right)$ for $\ell=0, \ldots, L-1, \overline{\bar{g}}_{n, L} \approx Y\left(t_{n}\right), \overline{\bar{g}}_{n, L+1} \approx Z\left(t_{n}\right)$ by setting first

$$
\overline{\bar{g}}_{0, \ell} \equiv 0, \quad \ell=0, \ldots, L+1
$$

and, then recursively, for $n=0, \ldots, N-1$, define

$$
\begin{aligned}
\overline{\bar{g}}_{n+1, \ell}=\overline{\bar{g}}_{n, \ell}+\Delta t_{n} \xi^{2}\left(\overline{\bar{g}}_{n, \ell_{n}}+f_{0}\left(t_{n}\right)\right) \widetilde{\lambda}\left(t_{n}, \tau_{\ell}\right) & \\
& +\xi\left(\overline{\bar{g}}_{n, \ell_{n}}+f_{0}\left(t_{n}\right)\right) \lambda\left(t_{n}, \tau_{\ell}\right) \cdot \Delta W_{n}, \quad \ell=0, \ldots, L-1, \\
\overline{\bar{g}}_{n+1, L}= & \overline{\bar{g}}_{n, L}+\Delta t_{n}\left(\overline{\bar{g}}_{n, \ell_{n}}+f_{0}\left(t_{n}\right)\right), \\
\overline{\bar{g}}_{n+1, L+1}= & \overline{\bar{g}}_{n, L+1}+\Delta t_{n} F\left(\overline{\bar{g}}_{n, L}\right) U\left(\overline{\bar{g}}_{n, \ell_{n}}+f_{0}\left(t_{n}\right)\right)
\end{aligned}
$$

where the index $\ell_{n}$ has been defined in (2.4).

2.3. The Euler-Finite Element (EFE) method. The (EFE) method also approximates the $\tau$-function $g\left(t_{n}, \cdot\right)$, by a piecewise constant function $\overline{\bar{g}}\left(t_{n}, \cdot\right) \in S_{\Delta \tau}$, but is based in a variational formulation of (1.9)(1.10) with $S_{\Delta \tau}$ being the space of trial and test functions. In particular, the (EFE) is defined by the initial datum

$$
\overline{\bar{g}}_{0, \ell} \equiv 0, \quad \ell=0, \ldots, L+1,
$$

and, for $n=0, \ldots, N-1$, the recursion

$$
\begin{aligned}
\overline{\bar{g}}_{n+1, \ell}= & \overline{\bar{g}}_{n, \ell}+\Delta t_{n} \xi^{2}\left(\overline{\bar{g}}_{n, \ell_{n}}+f_{0}\left(t_{n}\right)\right) \Pi \widetilde{\lambda}\left(t_{n} ; \tau_{\ell}\right) \\
& \quad+\xi\left(\overline{\bar{g}}_{n, \ell_{n}}+f_{0}\left(t_{n}\right)\right) \Pi \lambda\left(t_{n} ; \tau_{\ell}\right) \cdot \Delta W_{n}, \quad \ell=0, \ldots, L-1, \\
\overline{\bar{g}}_{n+1, L}= & \overline{\bar{g}}_{n, L}+\Delta t_{n}\left(\overline{\bar{g}}_{n, \ell_{n}}+f_{0}\left(t_{n}\right)\right), \\
\overline{\bar{g}}_{n+1, L+1}= & \overline{\bar{g}}_{n, L+1}+\Delta t_{n} F\left(\overline{\bar{g}}_{n, L}\right) U\left(\overline{\bar{g}}_{n, \ell_{n}}+f_{0}\left(t_{n}\right)\right)
\end{aligned}
$$

where the index $\ell_{n}$ has been defined in (2.4).

2.4. Approximation of the quantity of interest $\mathbb{E}[\mathcal{G}(g)]$. The numerical approximation of $\mathcal{G}(g)$ defined in (1.11) involves both an approximation of the processes $g, Y, Z$, by computable quantities, and an approximation of the $\tau$-integral in (1.6).

To construct an approximation of $\Lambda\left(\Psi\left(g\left(t_{\max }, \cdot\right)+f_{0}(\cdot)\right)\right)$ we apply a composite quadrature formula, over the partition of $\left[0, \tau_{\max }\right]$, based on a quadrature rule $Q: C[0,1] \rightarrow \mathbb{R}$ with $N_{Q}$ nodes $s_{Q}=\left(s_{Q, i}\right)_{i=1}^{N_{Q}}$ and weights $w_{Q}=\left(w_{Q, i}\right)_{i=1}^{N_{Q}}$, i.e., for $v \in C([0,1] ; \mathbb{R})$ the quantity $Q(v)=\sum_{i=1}^{N_{Q}} w_{Q, i} v\left(s_{Q, i}\right)$ approximates the integral $\int_{0}^{1} v(x) d x$. Also, we assume that the quadrature rule $Q$ is of order $p_{Q}$, i.e., it is exact for polynomials of order less or equal to $p_{Q}-1$. For example, the Simpson rule has $N_{Q}=3, s_{Q}=\left(0, \frac{1}{2}, 1\right)$ and $w_{Q}=\left(\frac{1}{6}, \frac{2}{3}, \frac{1}{6}\right)$, with $p_{Q}=4$. Another example is the Gaussian quadrature with $N_{Q}=2, s_{Q}=$ $\left(\frac{1}{2}-\frac{1}{2 \sqrt{3}}, \frac{1}{2}+\frac{1}{2 \sqrt{3}}\right), w_{Q}=\left(\frac{1}{2}, \frac{1}{2}\right)$ and $p_{Q}=4$. We note that it is well known from the mathematical analysis of numerical quadrature that in general we have $p_{Q} \leq 2 N_{Q}$, and the maximum value $p_{Q}=2 N_{Q}$ is achieved only by the Gaussian quadrature.

Thus, for a fixed realization of $\overline{\bar{g}}$ obtained by the (EFD) or the (EFE) method, first we approximate $\Lambda_{\Psi}(g):=\Lambda\left(\Psi\left(g\left(t_{\max }, \cdot\right)+f_{0}(\cdot)\right)\right)$ by $\Lambda_{\Psi}(\overline{\bar{g}})=\Lambda\left(\Psi\left(\overline{\bar{g}}\left(t_{\max }, \cdot\right)+f_{0}(\cdot)\right)\right)$ and then we apply the composite quadrature formula to construct an approximation $\bar{\Lambda}_{\Psi, Q}(\overline{\bar{g}})$ of $\Lambda_{\Psi}(\overline{\bar{g}})$ as follows

$$
\begin{aligned}
\bar{\Lambda}_{\Psi, Q}(\overline{\bar{g}}) & =\sum_{\ell=\ell_{a}}^{L-1} \Delta \tau_{\ell} Q\left(\Psi\left(\overline{\bar{g}}\left(t_{\max }, \tau_{\ell}+\cdot \Delta \tau_{\ell}\right)+f_{0}\left(\tau_{\ell}+\cdot \Delta \tau_{\ell}\right)\right)\right) \\
& =\sum_{\ell=\ell_{a}}^{L-1} \Delta \tau_{\ell}\left[\sum_{i=1}^{N_{Q}} w_{Q, i} \Psi\left(\overline{\bar{g}}_{N, \ell}+f_{0}\left(\tau_{\ell}+s_{Q, i} \Delta \tau_{\ell}\right)\right)\right] .
\end{aligned}
$$

Note that $\overline{\bar{g}}\left(t_{\max }, \cdot\right)$ is piecewise constant over the partition of $\left[0, \tau_{\max }\right]$ and numerical quadrature error in (2.9) is caused only from the presence of the initial datum $f_{0}$. In particular, if the initial datum for 
the term structure, $f_{0}$, is a piecewise constant function on the maturity time partition, then there is no quadrature error. Finally, an approximation $\overline{\mathcal{G}}(\overline{\bar{g}})$ of $\mathcal{G}(g)$ is computed by

$$
\overline{\mathcal{G}}(\overline{\bar{g}}) \equiv F\left(\overline{\bar{g}}_{N, L}\right) G\left(\bar{\Lambda}_{\Psi, Q}(\overline{\bar{g}})\right)+\overline{\bar{g}}_{N, L+1} .
$$

The Monte Carlo method, [16, approximates the expectation of a given random variable $X$ by a sample average of $M$ independent realizations of $X$, i.e. $\mathbb{E}[X] \approx \mathcal{A}(M ; X) \equiv \frac{1}{M} \sum_{j=1}^{M} X\left(\omega_{j}\right)$. In particular, here we approximate $\mathbb{E}[\mathcal{G}(g)]$ by a sample average of $\overline{\mathcal{G}}(\overline{\bar{g}})$,

$$
\mathcal{A}(M ; \overline{\mathcal{G}}(\overline{\bar{g}})) \equiv \frac{1}{M} \sum_{j=1}^{M}\left[F\left(\overline{\bar{g}}_{N, L}\left(\omega_{j}\right)\right) G\left(\bar{\Lambda}_{\Psi, Q}\left(\overline{\bar{g}}\left(\omega_{j}\right)\right)\right)+\overline{\bar{g}}_{N, L+1}\left(\omega_{j}\right)\right] .
$$

Therefore, the exact computational weak error

$$
\mathcal{E}_{c} \equiv \mathbb{E}[\mathcal{G}(g)]-\mathcal{A}(M ; \overline{\mathcal{G}}(\overline{\bar{g}}))
$$

naturally separates into three error contributions as follows:

$$
\mathcal{E}_{c}=E_{D}+E_{Q}+E_{S}
$$

with

$$
\begin{aligned}
E_{D} \equiv \mathbb{E}[\mathcal{G}(g)]-\mathbb{E}[\mathcal{G}(\overline{\bar{g}})], \quad E_{Q} \equiv \mathbb{E}[\mathcal{G}(\overline{\bar{g}})]-\mathbb{E}[\overline{\mathcal{G}}(\overline{\bar{g}})] \\
E_{S} \equiv \mathbb{E}[\overline{\mathcal{G}}(\overline{\bar{g}})]-\mathcal{A}(M ; \overline{\mathcal{G}}(\overline{\bar{g}}))
\end{aligned}
$$

where $E_{D}$ is the error contribution from $t$ - and $\tau$ - discretization, $E_{Q}$ is the quadrature error in (2.9), and $E_{S}$ is the statistical error.

\section{Strong Convergence}

To carry out an error analysis for the numerical methods proposed in Section 2, we assume that there exists nonnegative constants $C_{\xi, 1}$ and $C_{\xi, 2}$ such that

$$
\left|\xi^{2}(x)\right| \leq C_{\xi, 1}(1+|x|) \quad \forall x \in \mathbb{R},
$$

and

$$
\left|\xi^{2}(x)-\xi^{2}(z)\right|+|\xi(x)-\xi(z)| \leq C_{\xi, 2}|x-z|, \quad \forall x, z \in \mathbb{R} .
$$

3.1. Bounds for moments. In Lemmas 3.1 and 3.2, we show, respectively, boundness for the moments of the $\tau$-derivatives of the solution $g$ to the problem (1.9)-(1.10), and for the functional value $\mathcal{G}(g)$.

Lemma 3.1. Let $D_{\star} \equiv\left[0, t_{\max }\right] \times\left[0, \tau_{\max }\right], g$ be the solution of (1.9)-(1.10) and $\nu \in \mathbb{N}_{0}$. Also, we assume that the derivatives $\partial_{\tau}^{\ell} \widetilde{\lambda}$ and $\left(\partial_{\tau}^{\ell} \lambda_{j}\right)_{j=1}^{J}$ are well defined and continuous on $D_{\star}$, for $\ell=0, \ldots, \nu$. Then, for $\ell=0, \ldots, \nu$ and $\kappa \in \mathbb{N}$, there exists a positive constant $C_{\kappa, \ell}^{M}$, depending on $\kappa, \ell,\left(\partial_{\tau}^{\ell} \lambda_{j}\right)_{j=1}^{J}$, $\partial_{\tau}^{\ell} \tilde{\lambda}, f_{0}, C_{\xi, 1}, \tau_{\max }$ and $t_{\max }$, such that

$$
\max _{(t, \tau) \in D_{\star}} \mathbb{E}\left[\left|\partial_{\tau}^{\ell} g(t, \tau)\right|^{2 \kappa}\right] \leq C_{\kappa, \ell}^{M},
$$

where $C_{\xi, 1}$ is the constant in (3.1).

Proof. Let $\kappa \in \mathbb{N}, \ell \in\{0, \ldots, \nu\}$ and $(t, \tau) \in D_{\star}$. Also, in order to simplify the notation, we set $t_{\star}:=t_{\max }$ and $\tau_{\star}:=\tau_{\max }$. Our first step is to use (1.9) to get

$$
\mathbb{E}\left[\left|\partial_{\tau}^{\ell} g(t, \tau)\right|^{2 \kappa}\right] \leq(J+1)^{2 \kappa-1}\left[T_{1, \kappa}^{\ell}(t, \tau)+T_{2, \kappa}^{\ell}(t, \tau)\right],
$$

where

$$
\begin{aligned}
& T_{1, \kappa}^{\ell}(t, \tau) \equiv \mathbb{E}\left[\left(\int_{0}^{t} \partial_{\tau}^{\ell} \widetilde{\lambda}(s, \tau) \xi^{2}\left(g(s, s)+f_{0}(s)\right) d s\right)^{2 \kappa}\right] \\
& T_{2, \kappa}^{\ell}(t, \tau) \equiv \sum_{j=1}^{J} \mathbb{E}\left[\left(\int_{0}^{t} \partial_{\tau}^{\ell} \lambda_{j}(s, \tau) \xi\left(g(s, s)+f_{0}(s)\right) d W^{j}(s)\right)^{2 \kappa}\right] .
\end{aligned}
$$


Using (3.1) and applying the Hölder inequality we have

$$
\begin{aligned}
& T_{1, \kappa}^{\ell}(t, \tau) \leq \leq\left(C_{\xi, 1}\right)^{2 \kappa} \mathbb{E}\left[\left(\int_{0}^{t}\left|\partial_{\tau}^{\ell} \widetilde{\lambda}(s, \tau)\right|\left(1+\left|f_{0}(s)\right|+|g(s, s)|\right) d s\right)^{2 \kappa}\right] \\
& \leq 2^{2 \kappa-1}\left(C_{\xi, 1}\right)^{2 \kappa} \mathbb{E}\left[\left(\int_{0}^{t}\left|\partial_{\tau}^{\ell} \widetilde{\lambda}(s, \tau)\right|\left(1+\left|f_{0}(s)\right|\right) d s\right)^{2 \kappa}\right.\left.+\left(\int_{0}^{t}\left|\partial_{\tau}^{\ell} \widetilde{\lambda}(s, \tau)\right||g(s, s)| d s\right)^{2 \kappa}\right] \\
& \leq C_{1}^{\kappa, \ell}+C_{2}^{\kappa, \ell} \int_{0}^{t} \mathbb{E}\left[(g(s, s))^{2 \kappa}\right] d s,
\end{aligned}
$$

where $C_{1}^{\kappa, \ell} \equiv 2^{2 \kappa-1}\left(C_{\xi, 1}\right)^{2 \kappa} \max _{\tau \in\left[0, \tau_{\star}\right]}\left(\int_{0}^{t_{\star}}\left|\partial_{\tau}^{\ell} \widetilde{\lambda}(s, \tau)\right|\left(1+\left|f_{0}(s)\right|\right) d s\right)^{2 \kappa}$ and

$$
C_{2}^{\kappa, \ell} \equiv 2^{2 \kappa-1}\left(C_{\xi, 1}\right)^{2 \kappa} \max _{\tau \in\left[0, \tau_{\star}\right]}\left(\int_{0}^{t_{\star}}\left|\partial_{\tau}^{\ell} \widetilde{\lambda}(s, \tau)\right|^{\frac{2 \kappa}{2 \kappa-1}} d s\right)^{2 \kappa-1} .
$$

Next, using the properties of the Itô integral and (3.1), we obtain

$$
\begin{aligned}
T_{2, \kappa}^{\ell}(t, \tau) & \leq(2 \kappa-1) ! ! \sum_{j=1}^{J}\left(\int_{0}^{t}\left(\partial_{\tau}^{\ell} \lambda_{j}(s, \tau)\right)^{2} \mathbb{E}\left[\xi^{2}\left(g(s, s)+f_{0}(s)\right)\right] d s\right)^{\kappa} \\
& \leq(2 \kappa-1) ! !\left(C_{\xi, 1}\right)^{\kappa} \sum_{j=1}^{J}\left(\int_{0}^{t}\left(\partial_{\tau}^{\ell} \lambda_{j}(s, \tau)\right)^{2}\left(1+\left|f_{0}(s)\right|+\mathbb{E}[|g(s, s)|]\right) d s\right)^{\kappa} \\
& \leq(2 \kappa-1) ! !\left(C_{\xi, 1}\right)^{\kappa} \sum_{j=1}^{J}\left(\int_{0}^{t}\left(\partial_{\tau}^{\ell} \lambda_{j}(s, \tau)\right)^{2}\left(2+\left|f_{0}(s)\right|+\mathbb{E}\left[|g(s, s)|^{2}\right]\right) d s\right)^{\kappa} \\
& \leq C_{4}^{\kappa, \ell}+C_{3}^{\kappa, \ell}\left(\int_{0}^{t} \mathbb{E}\left[|g(s, s)|^{2}\right] d s\right)^{\kappa},
\end{aligned}
$$

where $C_{3}^{\kappa, \ell} \equiv(2 \kappa-1) ! ! 2^{\kappa-1}\left(C_{\xi, 1}\right)^{\kappa}\left(\sum_{j=1}^{J} \max _{D_{\star}}\left|\partial_{\tau}^{\ell} \lambda_{j}\right|^{2 \kappa}\right)$ and $C_{4}^{\kappa, \ell} \equiv C_{3}^{\kappa, \ell}\left(\int_{0}^{t_{\max }}\left(2+\left|f_{0}(s)\right|\right) d s\right)^{\kappa}$. Now, combine (3.4), (3.5) and (3.6), to arrive at

$$
\mathbb{E}\left[\left(\partial_{\tau}^{\ell} g(t, \tau)\right)^{2 \kappa}\right] \leq C_{I}^{\kappa, \ell}+C_{I I}^{\kappa, \ell}\left(\int_{0}^{t} \mathbb{E}\left[|g(s, s)|^{2}\right] d s\right)^{\kappa}+C_{I I I}^{\kappa, \ell} \int_{0}^{t} \mathbb{E}\left[|g(s, s)|^{2 \kappa}\right] d s,
$$

where $C_{I}^{\kappa, \ell}=(J+1)^{2 \kappa-1}\left(C_{1}^{\kappa, \ell}+C_{4}^{\kappa, \ell}\right), C_{I I}^{\kappa \ell}=(J+1)^{2 \kappa-1} C_{2}^{\kappa, \ell}$ and $C_{I I}^{\kappa, \ell}=(J+1)^{2 \kappa-1} C_{3}^{\kappa, \ell}$.

Consider the case $\kappa=1$ and $\ell=0$, and set $\tau=t$ in (3.7), to obtain

$$
\mathbb{E}\left[|g(t, t)|^{2}\right] \leq C_{I}^{1,0}+\left(C_{I I}^{1,0}+C_{I I I}^{1,0}\right) \int_{0}^{t} \mathbb{E}\left[|g(s, s)|^{2}\right] d s, \quad \forall t \in\left[0, t_{\star}\right],
$$

which, after the application of the Grönwall lemma, yields

$$
\mathbb{E}\left[|g(t, t)|^{2}\right] \leq C_{I}^{1,0} e^{\left(C_{I I}^{1,0}+C_{I I}^{1,0}\right) t}, \quad \forall t \in\left[0, t_{\star}\right] .
$$

Now, combine (3.8) and (3.7) (with $\kappa=1$ ), to get

$$
\mathbb{E}\left[\left|\partial_{\tau}^{\ell} g(t, \tau)\right|^{2}\right] \leq C_{I}^{1, \ell}+\frac{\left(C_{I I}^{1, \ell}+C_{I I I}^{1, \ell}\right) C_{I}^{1,0}}{C_{I I}^{1,0}+C_{I I}^{1,0}}\left[e^{\left(C_{I I}^{1,0}+C_{I I}^{1,0}\right) t}-1\right], \quad \forall(t, \tau) \in D_{\star},
$$

for $\ell=0, \ldots, \nu$, which establishes (3.3) for $\kappa=1$.

Now, consider the case $\kappa \geq 2$. Then, use (3.8) and (3.7), to obtain

$$
\mathbb{E}\left[\left|\partial_{\tau}^{\ell} g(t, \tau)\right|^{2 \kappa}\right] \leq C_{I V}^{\kappa, \ell}+C_{I I}^{\kappa, \ell} \int_{0}^{t} \mathbb{E}\left[|g(s, s)|^{2 \kappa}\right] d s, \quad \forall(t, \tau) \in D_{\star}, \quad \ell=0, \ldots, \nu,
$$


where $C_{I V}^{\kappa, \ell}=C_{I}^{\kappa, \ell}+C_{I I}^{\kappa, \ell}\left[\frac{C_{I}^{1,0}}{C_{I I}^{1,0}+C_{I I}^{1,0}}\left(e^{\left(C_{I I}^{1,0}+C_{I I}^{1,0}\right) t_{\max }}-1\right)\right]^{\kappa}$. Take $\ell=0$ and set $\tau=t$ in (3.10), to obtain

$$
\mathbb{E}\left[|g(t, t)|^{2 \kappa}\right] \leq C_{I V}^{\kappa, 0}+C_{I I I}^{\kappa, 0} \int_{0}^{t} \mathbb{E}\left[|g(s, s)|^{2 \kappa}\right] d s, \quad \forall t \in\left[0, t_{\star}\right] .
$$

Apply again the Grönwall lemma, to conclude that

$$
\mathbb{E}\left[|g(t, t)|^{2 \kappa}\right] \leq C_{I V}^{\kappa, 0} e^{C_{I I}^{\kappa, 0} t}, \quad \forall t \in\left[0, t_{\star}\right] .
$$

Finally, combine (3.11) and (3.10) to have

$$
\mathbb{E}\left[\left|\partial_{\tau}^{\ell} g(t, \tau)\right|^{2 \kappa}\right] \leq C_{I V}^{\kappa, \ell}+\frac{C_{I I I}^{\kappa, \ell} C_{N V}^{\kappa, 0}}{C_{I I}^{\kappa, 0}}\left(e^{C_{I I}^{\kappa, 0} t}-1\right), \quad \forall(t, \tau) \in D_{\star}, \quad \ell=0, \ldots, \nu,
$$

which yields the desired bound (3.3) for $\kappa \geq 2$.

Lemma 3.2. Let $(g, Y, Z)$ be the solution of the system (1.9)-(1.10). Also, we assume that the functions $F, G, \Psi, U: \mathbb{R} \rightarrow \mathbb{R}$ have polynomial growth $p_{F}, p_{G}, p_{\Psi}$ and $p_{U}$ with constants $C_{F}, C_{G}, C_{\Psi}$ and $C_{U}$, respectively. Then, for $\kappa \in \mathbb{N}$, there exists a positive constant $C_{\kappa}$, depending on $\kappa$ and the data of the problem, such that

$$
\mathbb{E}\left[\left|F\left(Y\left(t_{\max }\right)\right)\right|^{2 \kappa}\right]+\mathbb{E}\left[\left|G\left(\Lambda\left(\Psi\left(g\left(t_{\max }, \cdot\right)+f_{0}\right)\right)\right)\right|^{2 \kappa}\right]+\mathbb{E}\left[\left|Z\left(t_{\max }\right)\right|^{2 \kappa}\right] \leq C_{\kappa} .
$$

Proof. Let $\kappa \in \mathbb{N}$. To simplify the notation, we set $\tau_{\star}:=\tau_{\max }, t_{\star}:=t_{\max }$ and $\Upsilon(\tau):=g\left(t_{\max }, \tau\right)+f_{0}(\tau)=$ $f\left(t_{\max }, \tau\right)$ for $\tau \in\left[0, \tau_{\max }\right]$. Since $F, U$ and $G$ have polynomial growth, using the Hölder inequality and (3.3) for $\ell=0$, we obtain

and

$$
\begin{gathered}
\mathbb{E}\left[|F(Y(t))|^{2 \kappa}\right] \leq\left(C_{F}\right)^{2 \kappa} 2^{2 \kappa-1}\left(1+\mathbb{E}\left[|Y(t)|^{2 \kappa p_{F}}\right]\right), \quad \forall t \in\left[0, t_{\star}\right], \\
\mathbb{E}\left[|Y(t)|^{2 m}\right] \leq\left(2 t_{\star}\right)^{2 m-1} \int_{0}^{t}\left(\mathbb{E}\left[|g(s, s)|^{2 m}\right]+\left|f_{0}(s)\right|^{2 m}\right) d s \\
\leq\left(2 t_{\star}\right)^{2 m-1} \int_{0}^{t_{\star}}\left(C_{2 m, 0}^{M}+\left|f_{0}(s)\right|^{2 m}\right) d s, \quad \forall t \in\left[0, t_{\star}\right], \quad \forall m \in \mathbb{N}, \\
\mathbb{E}\left[\left|Z\left(t_{\star}\right)\right|^{2 \kappa}\right] \leq\left(t_{\star}\right)^{2 \kappa-1}\left\{\int_{0}^{t_{\star}} \mathbb{E}\left[|F(Y(s))|^{4 \kappa}\right] d s+\int_{0}^{t_{\star}} \mathbb{E}\left[|U(f(s, s))|^{4 \kappa}\right] d s\right\}, \\
\mathbb{E}\left[|U(f(t, t))|^{2 m}\right] \leq\left(C_{U}\right)^{2 m} 3^{2 m-1}\left(1+\left|f_{0}(t)\right|^{2 m p_{U}}+\mathbb{E}\left[|g(t, t)|^{2 m p_{U}}\right]\right) \\
\leq\left(C_{U}\right)^{2 m} 3^{2 m-1}\left(1+\left|f_{0}(t)\right|^{2 m p_{U}}+C_{2 m p_{U}, 0}^{M}\right), \quad \forall t \in\left[0, t_{\star}\right], \quad \forall m \in \mathbb{N}, \\
\mathbb{E}\left[|G(\Lambda(\Psi(\Upsilon)))|^{2 \kappa}\right] \leq\left(C_{G}\right)^{2 \kappa}\left(1+\mathbb{E}\left[|\Lambda(\Psi(\Upsilon))|^{2 \kappa p_{G}}\right]\right), \\
\mathbb{E}\left[|\Lambda(\Psi(\Upsilon))|^{2 m}\right] \leq\left(\tau_{\star}-\tau_{a}\right)^{2 m-1} \int_{\tau_{a}}^{\tau_{\max }} \mathbb{E}\left[|\Psi(\Upsilon(\tau))|^{2 m}\right] d \tau, \quad \forall m \in \mathbb{N},
\end{gathered}
$$

$$
\begin{aligned}
\mathbb{E}\left[|\Psi(\Upsilon(\tau))|^{2 m}\right] & \leq\left(C_{\Psi}\right)^{2 m} 3^{2 m-1}\left(1+\mathbb{E}\left[\left|g\left(t_{\star}, \tau\right)\right|^{2 m p_{\Psi}}\right]+\left|f_{0}(\tau)\right|^{2 m p_{\Psi}}\right) \\
& \leq\left(C_{\Psi}\right)^{2 m} 3^{2 m-1}\left(1+\left|f_{0}(\tau)\right|^{2 m p_{\Psi}}+C_{2 m p_{\Psi}, 0}^{M}\right), \quad \forall \tau \in\left[0, \tau_{\star}\right], \quad \forall m \in \mathbb{N} .
\end{aligned}
$$

Thus, we obtain (3.12) combining the inequalities (3.13)-(3.19) above.

In Lemma 3.3 below, we show boundness for the moments of the numerical approximations produced by the (EFD) and the (EFM) method.

Lemma 3.3. Let $\mathcal{I}:=\{0, \ldots, N\} \times\{0, \ldots, L-1\}$ and $\left(\overline{\bar{g}}_{n, \ell}\right)_{(n, \ell) \in \mathcal{I}}$ be the numerical approximations produced by the (EFD) or the (EFM) method. Then, for $\kappa \in \mathbb{N}$, there exists a nonnegative constant $C_{D, \kappa}^{M}$, depending on $\kappa,\left(\lambda_{j}\right)_{j=1}^{J}, \widetilde{\lambda}, f_{0}, C_{\xi, 1}, \tau_{\max }$ and $t_{\max }$, such that

$$
\max _{(n, \ell) \in \mathcal{I}} \mathbb{E}\left[\left|\overline{\bar{g}}_{n, \ell}\right|^{2 \kappa}\right] \leq C_{D, \kappa}^{M},
$$

where $C_{\xi, 1}$ is the constant in (3.1). 
Proof. Let $D_{\star} \equiv\left[0, t_{\max }\right] \times\left[0, \tau_{\max }\right], \kappa \in \mathbb{N},(n, \ell) \in \mathcal{I}$ with $n \geq 1$. Then, from (2.6) and (2.8), we conclude that

$$
\overline{\bar{g}}_{n, \ell}=\sum_{m=0}^{n-1} \Delta t_{m} \xi^{2}\left(\overline{\bar{g}}_{m, \ell_{m}}+f_{0}^{m}\right) \nu^{m, \ell}+\sum_{m=0}^{n-1} \sum_{j=1}^{J} \xi\left(\overline{\bar{g}}_{m, \ell_{m}}+f_{0}^{m}\right) \mu_{j}^{m, \ell} \Delta W_{m}^{j},
$$

where $f_{0}^{m}:=f_{0}\left(t_{m}\right), \nu^{m, \ell}=\widetilde{\lambda}\left(t_{m}, \tau_{\ell}\right)$ and $\mu_{j}^{m, \ell}=\lambda_{j}\left(t_{m}, \tau_{\ell}\right)$ for the (EFD) method, and $\nu^{m, \ell}=\Pi \widetilde{\lambda}\left(t_{m} ; \tau_{\ell}\right)$ and $\mu_{j}^{m, \ell}=\Pi \lambda_{j}\left(t_{m} ; \tau_{\ell}\right)$ for the (EFE) method. Thus, we obtain

$$
\mathbb{E}\left[\left|\overline{\bar{g}}_{n, \ell}\right|^{2 \kappa}\right] \leq(J+1)^{2 \kappa-1}\left(T_{1, \kappa}^{n, \ell}+T_{2, \kappa}^{n, \ell}\right)
$$

where

$$
\begin{aligned}
& T_{1, \kappa}^{n, \ell}:=\mathbb{E}\left[\left(\sum_{m=0}^{n-1} \Delta t_{m} \nu^{m, \ell} \xi^{2}\left(\overline{\bar{g}}_{m, \ell_{m}}+f_{0}^{m}\right)\right)^{2 \kappa}\right], \\
& T_{2, \kappa}^{n, \ell}:=\sum_{j=1}^{J} \mathbb{E}\left[\left(\sum_{m=0}^{n-1} \mu_{j}^{m, \ell} \xi\left(\overline{\bar{g}}_{m, \ell_{m}}+f_{0}^{m}\right) \Delta W_{m}^{j}\right)^{2 \kappa}\right] .
\end{aligned}
$$

Using (3.1) we bound $T_{1, \kappa}^{n, \ell}$ as follows

$$
\begin{aligned}
T_{1, \kappa}^{n, \ell} & \leq\left(C_{\xi, 1}\right)^{2 \kappa} \mathbb{E}\left[\left(\sum_{m=0}^{n-1} \Delta t_{m}\left|\nu^{m, \ell}\right|\left(1+\left|f_{0}^{m}\right|+\left|\overline{\bar{g}}_{m, \ell_{m}}\right|\right)\right)^{2 \kappa}\right] \\
& \leq 2^{2 \kappa-1}\left(C_{\xi, 1}\right)^{2 \kappa} \mathbb{E}\left[\left(\sum_{m=0}^{n-1} \Delta t_{m}\left|\nu^{m, \ell}\right|\left(1+\left|f_{0}^{m}\right|\right)\right)^{2 \kappa}+\left(\sum_{m=0}^{n-1} \Delta t_{m}\left|\nu^{m, \ell}\right|\left|\overline{\bar{g}}_{m, \ell_{m}}\right|\right)^{2 \kappa}\right]
\end{aligned}
$$

which, after applying the Hölder inequality, yields

$$
T_{1, \kappa}^{n, \ell} \leq C_{D, 1, \kappa}\left(t_{\max }+\sum_{m=0}^{n-1} \Delta t_{m} \mathbb{E}\left[\left|\overline{\bar{g}}_{m, \ell_{m}}\right|^{2 \kappa}\right]\right) .
$$

where $C_{D, 1, \kappa}=\left(2 C_{\xi, 1}\right)^{2 \kappa}\left(t_{\max }\right)^{2 \kappa-1} \max _{D_{\star}}\left[|\widetilde{\lambda}|\left(1+\left|f_{0}\right|\right)\right]^{2 \kappa}$. Also, using the properties of independent Gaussian random variables and (3.1), we obtain

$$
\begin{aligned}
T_{2}^{n, \ell} & \leq(2 \kappa-1) ! ! \sum_{j=1}^{J}\left(\sum_{m=0}^{n-1} \Delta t_{m}\left(\mu_{j}^{m, \ell}\right)^{2} \mathbb{E}\left[\xi^{2}\left(\overline{\bar{g}}_{m, \ell_{m}}+f_{0}^{m}\right)\right]\right)^{\kappa} \\
& \leq(2 \kappa-1) ! !\left(C_{\xi, 1}\right)^{\kappa} \max _{D_{\star}}|\lambda|^{2 \kappa}\left(\sum_{m=0}^{n-1} \Delta t_{m}\left(2+\left|f_{0}^{m}\right|+\mathbb{E}\left[\left|\overline{\bar{g}}_{m, \ell_{m}}\right|^{2}\right]\right)\right)^{\kappa} \\
& \leq(2 \kappa-1) ! !\left(C_{\xi, 1}\right)^{\kappa} \max _{D_{\star}}|\lambda|^{2 \kappa}\left[t_{\max } \max _{\left[0, t_{\max }\right]}\left(2+\left|f_{0}\right|\right)+\sum_{m=0}^{n-1} \Delta t_{m} \mathbb{E}\left[\left|\overline{\bar{g}}_{m, \ell_{m}}\right|^{2}\right]\right]^{\kappa}
\end{aligned}
$$

which yields that

$$
T_{2}^{n, \ell} \leq C_{D, 2, \kappa}\left[\left(t_{\max }\right)^{\kappa}+\left(\sum_{m=0}^{n-1} \Delta t_{m} \mathbb{E}\left[\left|\overline{\bar{g}}_{m, \ell_{m}}\right|^{2}\right]\right)^{\kappa}\right]
$$

where $C_{D, 2, \kappa}=2^{\kappa-1}(2 \kappa-1) ! !\left(C_{\xi, 1}\right)^{\kappa} \max _{D_{\star}}|\lambda|^{2 \kappa} \max _{\left[0, t_{\max }\right]}\left(2+\left|f_{0}\right|\right)^{\kappa}$. Now, combining (3.22), (3.23) and (3.24) we obtain

$$
\mathbb{E}\left[\left|\overline{\bar{g}}_{n, \ell}\right|^{2 \kappa}\right] \leq C_{\kappa}^{I, D}+C_{\kappa}^{I I, D} \sum_{m=0}^{n-1} \Delta t_{m} \mathbb{E}\left[\left|\overline{\bar{g}}_{m, \ell_{m}}\right|^{2 \kappa}\right]+C_{\kappa}^{I I I, D}\left(\sum_{m=0}^{n-1} \Delta t_{m} \mathbb{E}\left[\left|\overline{\bar{g}}_{m, \ell_{m}}\right|^{2}\right]\right)^{\kappa},
$$

where $C_{\kappa}^{I, D}, C_{\kappa}^{I I, D}$ and $C_{\kappa}^{I I I, D}$ are constants that depend on $J, \kappa, t_{\max }, C_{D, 1, \kappa}$ and $C_{D, 2, \kappa}$. 
First, let us consider the case $\kappa=1$. Then, setting $\ell=\ell_{n}$ in (3.25), we obtain

$$
\mathbb{E}\left[\left|\overline{\bar{g}}_{n, \ell_{n}}\right|^{2}\right] \leq C_{1}^{I, D}+C_{1}^{I V, D} \sum_{m=0}^{n-1} \Delta t_{m} \mathbb{E}\left[\left|\overline{\bar{g}}_{m, \ell_{m}}\right|^{2}\right], \quad n=1, \ldots, N,
$$

where $C_{1}^{I V, D}=C_{1}^{I I, D}+C_{1}^{I I I, D}$. Setting $\beta_{n}:=\frac{1}{C_{1}^{I, D}} \mathbb{E}\left[\left|\overline{\bar{g}}_{n, \ell_{n}}\right|^{2}\right]$ for $n=0, \ldots, N$, (3.26) is written equivalently as follows

$$
\beta_{n} \leq 1+C_{1}^{I V, D} \sum_{m=0}^{n-1} \Delta t_{m} \beta_{m}, \quad n=1, \ldots, N .
$$

Now, setting $\rho_{1}:=1$ and $\rho_{n}:=1+C_{1}^{I V, D} \sum_{m=1}^{n-1} \Delta t_{m} \rho_{m}$ for $n=2, \ldots, N$ and observing that $\beta_{0}=0$, we use (3.27) and apply a simple induction argument to get

$$
\beta_{n} \leq \rho_{n}, \quad n=1, \ldots, N .
$$

Since $\rho_{n}=\left(1+C_{1}^{I V, D} \Delta t_{n-1}\right) \rho_{n-1}$ for $n=2, \ldots, N$, we use the inequality $e^{x} \geq 1+x$ for $x \geq 0$, and a simple induction argument to conclude that

$$
\rho_{n} \leq \exp \left(C_{1}^{I, D} t_{n}\right), \quad n=1, \ldots, N .
$$

Thus, (3.28) and (3.29) yield

$$
\max _{0 \leq m \leq N} \mathbb{E}\left[\left|\overline{\bar{g}}_{m, \ell_{m}}\right|^{2}\right] \leq C_{1}^{I, D} \exp \left(C_{1}^{I V, D} t_{\max }\right)
$$

which, along with (3.25), establishes (3.20) for $\kappa=1$.

Now, we assume that $\kappa \geq 2$. Then, we combine (3.25) and (3.30) to obtain

$$
\mathbb{E}\left[\left|\overline{\bar{g}}_{n, \ell_{n}}\right|^{2 \kappa}\right] \leq C_{\kappa}^{V, D}+C_{\kappa}^{I I, D} \sum_{m=0}^{n-1} \Delta t_{m} \mathbb{E}\left[\left|\overline{\bar{g}}_{m, \ell_{m}}\right|^{2 \kappa}\right], \quad n=1, \ldots, N,
$$

where $C_{\kappa}^{V, D}=C_{\kappa}^{I, D}+C_{\kappa}^{I I I, D}\left(t_{\max }\right)^{\kappa}\left(C_{1}^{I, D} \exp \left(C_{1}^{I V, D} t_{\max }\right)\right)^{\kappa}$. Then, proceeding as in obtaining (3.30) from (3.26), we arrive at

$$
\max _{0 \leq m \leq N} \mathbb{E}\left[\left|\overline{\bar{g}}_{m, \ell_{m}}\right|^{2 \kappa}\right] \leq C_{1}^{V, D} \exp \left(C_{1}^{I I, D} t_{\max }\right),
$$

which, along with (3.25) and (3.30), yields (3.20) for $\kappa \geq 2$.

3.2. Estimates for the consistency error. In Lemmas 3.4 and 3.5 below, we show that some Lipschitztype properties for the solution $g$ to the problem (1.9)-(1.10) hold.

Lemma 3.4. Let $\kappa \in \mathbb{N}$ and $g$ be the solution of (1.9)-(1.10). Then, it holds that

$$
\mathbb{E}\left[\left|g\left(t, \tau_{1}\right)-g\left(t, \tau_{2}\right)\right|^{2 \kappa}\right] \leq C_{\kappa, 1}^{M}\left|\tau_{1}-\tau_{2}\right|^{2 \kappa}, \quad \forall \tau_{1}, \tau_{2} \in\left[0, \tau_{\max }\right], \quad \forall t \in\left[0, t_{\max }\right],
$$

where $C_{\kappa, 1}^{M}$ is the constant in (3.3) for $\ell=1$.

Proof. Let $t \in\left[0, t_{\max }\right]$ and $\tau_{1}, \tau_{2} \in\left[0, \tau_{\max }\right]$ with $\tau_{2} \geq \tau_{1}$. Then, applying the Hölder inequality, we have

$$
\begin{aligned}
\mathbb{E}\left[\left|g\left(t, \tau_{1}\right)-g\left(t, \tau_{2}\right)\right|^{2 \kappa}\right] & =\mathbb{E}\left[\left|\int_{\tau_{1}}^{\tau_{2}} \partial_{\tau} g(t, \tau) d \tau\right|^{2 \kappa}\right] \\
& \leq\left|\tau_{2}-\tau_{1}\right|^{2 \kappa-1} \int_{\tau_{1}}^{\tau_{2}} \mathbb{E}\left[\left|\partial_{\tau} g(t, \tau)\right|^{2 \kappa}\right] d \tau \\
& \leq\left|\tau_{2}-\tau_{1}\right|^{2 \kappa} \max _{\tau \in\left[\tau_{1}, \tau_{2}\right]} \mathbb{E}\left[\left|\partial_{\tau} g(t, \tau)\right|^{2 \kappa}\right] .
\end{aligned}
$$

Thus, we obtain (3.33) combining the inequality above and (3.3) for $\ell=1$.

Lemma 3.5. Let $\kappa \in \mathbb{N}$ and $g$ be the solution of (1.9)-(1.10). Then, there exists a nonnegative constant $C_{\mathrm{Lip}}$, depending on $\kappa, J, \lambda, \widetilde{\lambda}, f_{0}, C_{\xi, 1}, \tau_{\max }$ and $t_{\max }$, such that

$$
\mathbb{E}\left[\left|g\left(t_{1}, \tau\right)-g\left(t_{2}, \tau\right)\right|^{2 \kappa}\right] \leq C_{\text {Lip }}\left|t_{1}-t_{2}\right|^{\kappa}, \quad \forall t_{1}, t_{2} \in\left[0, t_{\max }\right], \quad \forall \tau \in\left[0, \tau_{\max }\right] .
$$


Proof. Let $D_{\star} \equiv\left[0, t_{\max }\right] \times\left[0, \tau_{\max }\right], \tau \in\left[0, \tau_{\max }\right]$ and $t_{1}, t_{2} \in\left[0, t_{\max }\right]$ with $t_{2} \geq t_{1}$. Proceeding as in the proof of Lemma 3.1 we obtain

$$
\mathbb{E}\left[\left|g\left(\tau, t_{1}\right)-g\left(\tau, t_{2}\right)\right|^{2 \kappa}\right] \leq(J+1)^{2 \kappa-1}\left[B_{\kappa, I}\left(\tau ; t_{1}, t_{2}\right)+B_{\kappa, I I}\left(\tau ; t_{1}, t_{2}\right)\right]
$$

where

$$
\begin{aligned}
& B_{\kappa, I}\left(\tau ; t_{1}, t_{2}\right)=\mathbb{E}\left[\left(\int_{t_{1}}^{t_{2}} \widetilde{\lambda}(s, \tau) \xi^{2}\left(g(s, s)+f_{0}(s)\right) d s\right)^{2 \kappa}\right] \\
& B_{\kappa, I I}\left(\tau ; t_{1}, t_{2}\right)=(2 \kappa-1) ! ! \sum_{j=1}^{J}\left(\int_{t_{1}}^{t_{2}}\left(\lambda_{j}(s, \tau)\right)^{2} \mathbb{E}\left[\xi^{2}\left(g(s, s)+f_{0}(s)\right)\right] d s\right)^{\kappa} .
\end{aligned}
$$

Using the Hölder inequality and (3.1) we obtain

$$
\begin{aligned}
B_{\kappa, I}\left(\tau ; t_{1}, t_{2}\right) & \leq\left(2 C_{\xi, 1}\right)^{2 \kappa} \max _{D_{\star}}|\widetilde{\lambda}|^{2 \kappa} \mathbb{E}\left[\left(\int_{t_{1}}^{t_{2}}\left(1+\left|f_{0}(s)\right|\right) d s\right)^{2 \kappa}+\left(\int_{t_{1}}^{t_{2}}|g(s, s)| d s\right)^{2 \kappa}\right] \\
& \leq\left(2 C_{\xi, 1}\right)^{2 \kappa} \max _{D_{\star}}|\widetilde{\lambda}|^{2}\left|t_{1}-t_{2}\right|^{2 \kappa}\left(\max _{s \in\left[t_{1}, t_{2}\right]}\left(1+\left|f_{0}(s)\right|\right)^{2 \kappa}+\max _{s \in\left[t_{1}, t_{2}\right]} \mathbb{E}\left[|g(s, s)|^{2 \kappa}\right]\right)
\end{aligned}
$$

and

$$
\begin{gathered}
B_{\kappa, I I}\left(\tau ; t_{1}, t_{2}\right) \leq(2 \kappa-1) ! !\left(C_{\xi, 1}\right)^{\kappa}\left(\sum_{j=1}^{J} \max _{D_{\star}}\left|\lambda_{j}\right|^{2 \kappa}\right)\left(\int_{t_{1}}^{t_{2}}\left(2+\left|f_{0}(s)\right|+\mathbb{E}\left[|g(s, s)|^{2}\right]\right) d s\right)^{\kappa} \\
\leq(2 \kappa-1) ! !\left(C_{\xi, 1}\right)^{\kappa}\left(\sum_{j=1}^{J} \max _{D_{\star}}\left|\lambda_{j}\right|^{2 \kappa}\right)\left|t_{2}-t_{1}\right|^{\kappa} \max _{s \in\left[t_{1}, t_{2}\right]}\left(2+\left|f_{0}(s)\right|+\mathbb{E}\left[|g(s, s)|^{2}\right]\right)^{\kappa} .
\end{gathered}
$$

Thus, (3.34) follows easily from (3.35), (3.36), (3.37) and (3.3) for $\ell=0$.

In Proposition 3.1 that follows, we prove a consistency result for the (EFD) and (EFE) methods defined in Section 2

Proposition 3.1. Let $\kappa \in \mathbb{N}, g$ be the solution of (1.9)-(1.10), $f_{0}^{m}:=f_{0}\left(t_{m}\right)$ for $m=0, \ldots, N$, and $\mathcal{K}_{n, \ell}$ be defined by

$$
\widehat{g}_{n+1, \ell}=\widehat{g}_{n, \ell}+\Delta t_{n} \nu^{n, \ell} \xi^{2}\left(\widehat{g}_{n, \ell_{n}}+f_{0}^{n}\right)+\sum_{j=1}^{J} \mu_{j}^{n, \ell} \xi\left(\widehat{g}_{n, \ell_{n}}+f_{0}^{n}\right) \Delta W_{n}^{j}+\mathcal{K}_{n, \ell},
$$

for $n=0, \ldots, N-1$ and $\ell=0, \ldots, L-1$, where $\nu^{n, \ell}=\widetilde{\lambda}\left(t_{n}, \tau_{\ell}\right), \mu_{j}^{n, \ell}=\lambda_{j}\left(t_{m}, \tau_{\ell}\right)$ and $\widehat{g}_{m, \ell}=g\left(t_{m}, \tau_{\ell}\right)$ for the (EFD) method, and $\nu^{m, \ell}=\Pi \widetilde{\lambda}\left(t_{m} ; \tau_{\ell}\right)$ and $\mu_{j}^{m, \ell}=\Pi \lambda_{j}\left(t_{m} ; \tau_{\ell}\right)$ and $\widehat{g}_{m, \ell}=\Pi g\left(t_{m} ; \tau_{\ell}\right)$ for the (EFE) method. Also, we assume that $f_{0} \in C^{1}\left(\left[0, \tau_{\max }\right] ; \mathbb{R}\right)$ and $\partial_{t} \widetilde{\lambda}, \partial_{t \tau} \widetilde{\lambda},\left(\partial_{t} \lambda_{j}\right)_{j=1}^{J},\left(\partial_{t \tau} \lambda_{j}\right)_{j=1}^{J}$ are well-defined and continuous on $\left[0, t_{\max }\right] \times\left[0, \tau_{\max }\right]$. Then, there exists a nonnegative constant $C_{c n, 1}$, independent of the partitions of the intervals $\left[0, t_{\max }\right]$ and $\left[0, \tau_{\max }\right]$, such that

$$
\mathbb{E}\left[\left|\sum_{m=0}^{n} \mathcal{K}_{m, \ell}\right|^{2 \kappa}\right] \leq C_{c n, 1}\left[(\Delta t)^{\kappa}+(\Delta \tau)^{2 \kappa}\right]
$$

for $n=0, \ldots, N-1$ and $\ell=0, \ldots, L-1$. In addition, for the (EFD) method there exists a nonnegative constant $C_{c n, 2}$, independent of the partitions of the intervals $\left[0, t_{\max }\right]$ and $\left[0, \tau_{\max }\right]$, such that

$$
\mathbb{E}\left[\left|\sum_{m=0}^{n} \mathcal{K}_{m, \ell+1}-\mathcal{K}_{m, \ell}\right|^{2 \kappa}\right] \leq C_{c n, 2}\left(\Delta \tau_{\ell}\right)^{2 \kappa}\left[(\Delta t)^{\kappa}+(\Delta \tau)^{2 \kappa}\right]
$$

for $n=0, \ldots, N-1$ and $\ell=0, \ldots, L-2$. 
Proof. Here, we set $D_{\star}:=\left[0, t_{\max }\right] \times\left[0, \tau_{\max }\right]$ and use the symbol $C$ for a generic constant independent of the partitions of the intervals $\left[0, t_{\max }\right]$ and $\left[0, \tau_{\max }\right]$. First, we observe that (1.9) yields that

$$
\begin{aligned}
\widehat{g}_{n+1, \ell}=\widehat{g}_{n, \ell} & +\int_{t_{n}}^{t_{n+1}} \nu^{\ell}(s) \xi^{2}\left(g(s, s)+f_{0}(s)\right) d s \\
& +\sum_{j=1}^{J} \int_{t_{n}}^{t_{n+1}} \mu_{j}^{\ell}(s) \xi\left(g(s, s)+f_{0}(s)\right) d W^{j}(s),
\end{aligned}
$$

for $n=0, \ldots, N-1$ and $\ell=0, \ldots, L-1$, where $\nu^{\ell}(s)=\widetilde{\lambda}\left(s, \tau_{\ell}\right)$ and $\mu_{j}^{\ell}(s)=\lambda_{j}\left(s, \tau_{\ell}\right)$ for the (EFD) method and $\nu^{\ell}(s)=\Pi \tilde{\lambda}\left(s ; \tau_{\ell}\right)$ and $\mu_{j}^{\ell}(s)=\Pi \lambda_{j}\left(s ; \tau_{\ell}\right)$ for the (EFE) method. Then, subtracting (3.41) from (3.38) we obtain $\sum_{m=0}^{n} \mathcal{K}_{m, \ell}=\sum_{i=1}^{4} E_{i, C}^{n, \ell}$ for $\ell=0, \ldots, L-1$ and $n=0, \ldots, N-1$, where

$$
\begin{aligned}
& E_{1, C}^{n, \ell} \equiv \sum_{m=0}^{n} \int_{t_{m}}^{t_{m+1}}\left(\nu^{\ell}(s)-\nu^{m, \ell}\right) \xi^{2}\left(g(s, s)+f_{0}(s)\right) d s, \\
& E_{2, C}^{n, \ell} \equiv \sum_{m=0}^{n} \int_{t_{m}}^{t_{m+1}} \nu^{m, \ell}\left[\xi^{2}\left(g(s, s)+f_{0}(s)\right)-\xi^{2}\left(\widehat{g}_{m, \ell_{m}}+f_{0}^{m}\right)\right] d s, \\
& E_{3, C}^{n, \ell} \equiv \sum_{j=1}^{J} \sum_{m=0}^{n} \int_{t_{m}}^{t_{m+1}}\left(\mu_{j}^{\ell}(s)-\mu_{j}^{n, \ell}\right) \xi\left(g(s, s)+f_{0}(s)\right) d W^{j}(s), \\
& E_{4, C}^{n, \ell} \equiv \sum_{j=1}^{J} \sum_{m=0}^{n} \int_{t_{m}}^{t_{m+1}} \mu_{j}^{n, \ell}\left[\xi\left(g(s, s)+f_{0}(s)\right)-\xi\left(\widehat{g}_{m, \ell_{m}}+f_{0}^{m}\right)\right] d W^{j}(s) .
\end{aligned}
$$

Next, using (3.1), the Hölder inequality and (3.3), we obtain

$$
\begin{aligned}
\mathbb{E}\left[\left(E_{1, C}^{n, \ell}\right)^{2 \kappa}\right] & \leq\left(C_{\xi, 1}\right)^{2 \kappa}(\Delta t)^{2 \kappa} \max _{D_{\star}}\left|\partial_{t} \widetilde{\lambda}\right|^{2 \kappa} \mathbb{E}\left[\left(\int_{0}^{t_{\max }}\left(1+\left|f_{0}(s)\right|+|g(s, s)|\right) d s\right)^{2 \kappa}\right] \\
& \leq C(\Delta t)^{2 \kappa} \mathbb{E}\left[\left(\int_{0}^{t_{\max }}\left(1+\left|f_{0}(s)\right| d s\right)^{2 \kappa}+\left(\int_{0}^{t_{\max }}|g(s, s)| d s\right)^{2 \kappa}\right]\right. \\
& \leq C(\Delta t)^{2 \kappa}\left[1+\left(t_{\max }\right)^{2 \kappa} \max _{s \in\left[0, t_{\max }\right]} \mathbb{E}\left[(g(s, s))^{2 \kappa}\right]\right] \\
& \leq C(\Delta t)^{2 \kappa}\left[1+\left(t_{\max }\right)^{2 \kappa} C_{\kappa, 0}^{M}\right]
\end{aligned}
$$

and

$$
\begin{aligned}
\mathbb{E}\left[\left(E_{3, C}^{n, \ell}\right)^{2 \kappa}\right] & \leq C \sum_{j=1}^{J} \mathbb{E}\left[\sum_{m=0}^{n} \int_{t_{m}}^{t_{m+1}}\left(\mu_{j}^{\ell}(s)-\mu_{j}^{m, \ell}\right) \xi\left(g(s, s)+f_{0}(s)\right) d W^{j}(s)\right]^{2 \kappa} \\
& \leq C \sum_{j=1}^{J}\left[\sum_{m=0}^{n} \int_{t_{m}}^{t_{m+1}}\left(\mu_{j}^{\ell}(s)-\mu_{j}^{m, \ell}\right)^{2} \mathbb{E}\left[\xi^{2}\left(g(s, s)+f_{0}(s)\right)\right] d s\right]^{\kappa} \\
& \leq C(\Delta t)^{2 \kappa}\left(\int_{0}^{t_{\max }}\left(1+\left|f_{0}(s)\right|+\mathbb{E}[|g(s, s)|]\right) d s\right)^{\kappa} \\
& \leq C(\Delta t)^{2 \kappa}\left(\int_{0}^{t_{\max }}\left(2+\left|f_{0}(s)\right|+\mathbb{E}\left[(g(s, s))^{2}\right]\right) d s\right)^{\kappa} \\
& \leq C(\Delta t)^{2 \kappa}\left(\int_{0}^{t_{\max }}\left(2+\left|f_{0}(s)\right|+C_{1,0}^{M}\right) d s\right)^{\kappa}
\end{aligned}
$$


Now, we apply (3.2) and the Hölder inequality, to get

$$
\begin{aligned}
\mathbb{E}\left[\left(E_{2, C}^{n, \ell}\right)^{2 \kappa}\right] & \leq C \mathbb{E}\left[\left(\sum_{m=0}^{n} \int_{t_{m}}^{t_{m+1}}\left(\left|g(s, s)-\widehat{g}_{m, \ell_{m}}\right|+\left|f_{0}(s)-f_{0}^{m}\right|\right) d s\right)^{2 \kappa}\right] \\
& \leq C \mathbb{E}\left[\left(\sum_{m=0}^{n} \int_{t_{m}}^{t_{m+1}}\left|f_{0}(s)-f_{0}^{m}\right| d s\right)^{2 \kappa}+\left(\sum_{m=0}^{n} \int_{t_{m}}^{t_{m+1}}\left|g(s, s)-\widehat{g}_{m, \ell_{m}}\right| d s\right)^{2 \kappa}\right] \\
& \leq C\left[\sum_{m=0}^{n} \int_{t_{m}}^{t_{m+1}}\left|f_{0}(s)-f_{0}^{m}\right|^{2 \kappa} d s+\sum_{m=0}^{n} \int_{t_{m}}^{t_{m+1}} \mathbb{E}\left[\left|g(s, s)-\widehat{g}_{m, \ell_{m}}\right|^{2 \kappa}\right] d s\right] \\
& \leq C\left[(\Delta t)^{2 \kappa} \max _{\left[0, \tau_{\max }\right]}\left|f_{0}^{\prime}\right|^{2 \kappa}+\sum_{m=0}^{n} \int_{t_{m}}^{t_{m+1}} \mathbb{E}\left[\left|g(s, s)-\widehat{g}_{m, \ell_{m}}\right|^{2 \kappa}\right] d s\right]
\end{aligned}
$$

and

$$
\begin{aligned}
\mathbb{E}\left[\left(E_{4, C}^{n, \ell}\right)^{2 \kappa}\right] & \leq C \sum_{j=1}^{J} \mathbb{E}\left[\sum_{m=0}^{n} \int_{t_{m}}^{t_{m+1}} \mu_{j}^{n, \ell}\left[\xi\left(g(s, s)+f_{0}(s)\right)-\xi\left(\widehat{g}_{m, \ell_{m}}+f_{0}^{m}\right)\right] d W^{j}(s)\right]^{2 \kappa} \\
& \leq C\left[\sum_{m=0}^{n} \int_{t_{m}}^{t_{m+1}}\left(\mu_{j}^{n, \ell}\right)^{2} \mathbb{E}\left[\left(\xi\left(g(s, s)+f_{0}(s)\right)-\xi\left(\widehat{g}_{m, \ell_{m}}+f_{0}^{m}\right)\right)^{2}\right] d s\right]^{\kappa} \\
& \leq C\left[\sum_{m=0}^{n} \int_{t_{m}}^{t_{m+1}}\left(\mathbb{E}\left[\left|g(s, s)-\widehat{g}_{m, \ell_{m}}\right|^{2}\right]+\left|f_{0}(s)-f_{0}^{m}\right|^{2}\right) d s\right]^{\kappa} \\
& \leq C\left[(\Delta t)^{2} \max _{\left[0, \tau_{\max }\right]}\left|f_{0}^{\prime}\right|^{2}+\sum_{m=0}^{n} \int_{t_{m}}^{t_{m+1}} \mathbb{E}\left[\left|g(s, s)-\widehat{g}_{m, \ell_{m}}\right|^{2}\right]\right]^{\kappa}
\end{aligned}
$$

Using (3.33), (3.34) and (2.4), we have

$$
\begin{aligned}
\mathbb{E}\left[\left|g(s, s)-\widehat{g}_{m, \ell_{m}}\right|^{2 \kappa}\right] \leq & C\left(\mathbb{E}\left[\left|g(s, s)-g\left(s, \tau_{\ell_{m}}\right)\right|^{2 \kappa}\right]+\mathbb{E}\left[\left|g\left(s, \tau_{\ell_{m}}\right)-g\left(t_{m}, \tau_{\ell_{m}}\right)\right|^{2 \kappa}\right]\right. \\
& \left.\quad+\mathbb{E}\left[\left|g\left(t_{m}, \tau_{\ell_{m}}\right)-\widehat{g}_{m, \ell_{m}}\right|^{2 \kappa}\right]\right) \\
\leq & C\left(\left|s-\tau_{\ell_{m}}\right|^{2 \kappa}+\left|s-t_{m}\right|^{\kappa}+\mathbb{E}\left[\left|g\left(t_{m}, \tau_{\ell_{m}}\right)-\widehat{g}_{m, \ell_{m}}\right|^{2 \kappa}\right]\right) \\
\leq & C\left(\left|s-t_{m}\right|^{2 \kappa}+\left|t_{m}-\tau_{\ell_{m}}\right|^{2 \kappa}+(\Delta t)^{\kappa}+\mathbb{E}\left[\left|g\left(t_{m}, \tau_{\ell_{m}}\right)-\widehat{g}_{m, \ell_{m}}\right|^{2 \kappa}\right]\right) \\
\leq & C\left(\left|\tau_{\ell_{m}+1}-\tau_{\ell_{m}}\right|^{2 \kappa}+(\Delta t)^{\kappa}+\mathbb{E}\left[\left|g\left(t_{m}, \tau_{\ell_{m}}\right)-\widehat{g}_{m, \ell_{m}}\right|^{2 \kappa}\right]\right) \\
\leq & C\left((\Delta \tau)^{2 \kappa}+(\Delta t)^{\kappa}+\mathbb{E}\left[\left|g\left(t_{m}, \tau_{\ell_{m}}\right)-\widehat{g}_{m, \ell_{m}}\right|^{2 \kappa}\right]\right)
\end{aligned}
$$

for $s \in\left[t_{m}, t_{m+1}\right]$ and $m=0, \ldots, N-1$. For the (EFE) method, after using (3.33), we have

$$
\begin{aligned}
\mathbb{E}\left[\left|g\left(t_{m}, \tau_{\ell_{m}}\right)-\widehat{g}_{m, \ell_{m}}\right|^{2 \kappa}\right] & \leq\left(\Delta \tau_{\ell_{m}}\right)^{-1} \int_{\tau_{\ell_{m}}}^{\tau_{\ell_{m}+1}} \mathbb{E}\left[\left(g\left(t_{m}, \tau_{\ell_{m}}\right)-g\left(t_{m}, \tau\right)\right)^{2 \kappa}\right] d \tau \\
& \leq C\left(\Delta \tau_{\ell_{m}}\right)^{-1} \int_{\tau_{\ell_{m}}}^{\tau_{\ell_{m}+1}}\left|\tau_{\ell_{m}}-\tau\right|^{2 \kappa} d \tau \\
& \leq C(\Delta \tau)^{2 \kappa}, \quad m=0, \ldots, N-1,
\end{aligned}
$$

while for the (EFD) method the term we estimate above vanishes. Finally, (3.46) and (3.47) yield

$$
\mathbb{E}\left[\left|g(s, s)-\widehat{g}_{m, \ell_{m}}\right|^{2 \kappa}\right] \leq C\left[(\Delta t)^{\kappa}+(\Delta \tau)^{2 \kappa}\right]
$$

for $s \in\left[t_{m}, t_{m+1}\right]$ and $m=0, \ldots, N-1$. Observing that $\mathbb{E}\left[\left(\sum_{\ell=0}^{n} \mathcal{K}_{m, \ell}\right)^{2 \kappa}\right] \leq 4^{2 \kappa-1} \sum_{i=1}^{4} \mathbb{E}\left[\left(E_{i, C}^{n, \ell}\right)^{2 \kappa}\right]$ for $\ell=0, \ldots, L-1$ and $n=0, \ldots, N-1$, and that estimate (3.48) holds for $\kappa=1$, the estimate (3.39) for the consistency error follows easily in view of (3.42), (3.43), (3.44), (3.45) and (3.48). 
Since, $\mathbb{E}\left[\left(\sum_{\ell=0}^{n} \mathcal{K}_{m, \ell+1}-\mathcal{K}_{m, \ell+1}\right)^{2 \kappa}\right] \leq 4^{2 \kappa-1} \sum_{i=1}^{4} \mathbb{E}\left[\left(E_{i, C}^{n, \ell+1}-\left(E_{i, C}^{n, \ell}\right)^{2 \kappa}\right]\right.$ for $\ell=0, \ldots, L-2$ and $n=0, \ldots, N-1$, we obtain (3.40) for the (EFD) nethod, observing that

$$
\begin{aligned}
\left|y^{m, \ell+1}-y^{m, \ell}\right| & \leq C \Delta \tau_{\ell} \\
\left|\left(y^{\ell+1}(s)-y^{m, \ell+1}\right)-\left(y^{\ell}(s)-y^{m, \ell}\right)\right| & =\left|\int_{t_{m}}^{s} \int_{\tau_{\ell}}^{\tau_{\ell+1}} \partial_{t \tau} y\left(t^{\prime}, \tau^{\prime}\right) d t^{\prime} d \tau^{\prime}\right| \\
& \leq C \Delta t \Delta \tau_{\ell}, \quad \forall s \in\left[t_{m}, t_{m+1}\right],
\end{aligned}
$$

where $y^{m, \ell}=\nu^{m, \ell}$ or $\mu_{j}^{n, \ell}$ and $y=\widetilde{\lambda}$ or $\lambda_{j}$, respectively, and proceeding as above.

3.3. Error estimation. In this section we derive an error estimate for the strong approximation error $\mathcal{G}(g)-\overline{\mathcal{G}}(\overline{\bar{g}})$ by splitting it as sum of the strong discretization error $\mathcal{G}(g)-\mathcal{G}(\overline{\bar{g}})$ which we estimate in Theorem 3.2 and of the strong mumerical quadrature error $\mathcal{G}(\overline{\bar{g}})-\overline{\mathcal{G}}(\overline{\bar{g}})$ which we estimate in Theorem 3.3 .

Theorem 3.2. Let $g, Y$ and $Z$ be the solution of (1.9)-1.10), $\mathcal{M}:=\{0, \ldots, N\} \times\{0, \ldots, L-2\}$ $\mathcal{J}:=\{0, \ldots, N\} \times\{0, \ldots, L-1\}, \mathcal{I}:=\{0, \ldots, N\} \times\{0, \ldots, L+1\}$ and $\left(\overline{\bar{g}}_{n, \ell}\right)_{(n, \ell) \in \mathcal{I}}$ be the numerical approximations produced by the (EFD) or the (EFM) method. Also, we assume that the functions $\Psi^{\prime}, F$, $F^{\prime}, G, G^{\prime}, U, U^{\prime}: \mathbb{R} \rightarrow \mathbb{R}$ have polynomial growth, and we define $\Lambda_{\Psi}(w):=\Lambda\left(\Psi\left(w+f_{0}\right)\right)$ for $w \in S_{\Delta \tau}$ or $w \in C\left(\left[0, \tau_{\max }\right] ; \mathbb{R}\right)$. Then, there exist nonnegative constants $\left(C_{i}^{C V}\right)_{i=1}^{6}$, independent of the partitions of the intervals $\left[0, t_{\max }\right]$ and $\left[0, \tau_{\max }\right]$, such that

$$
\begin{gathered}
\max _{(n, \ell) \in \mathcal{J}}\left(\mathbb{E}\left[\left|g\left(t_{n}, \tau_{\ell}\right)-\overline{\bar{g}}_{n, \ell}\right|^{2 \kappa}\right]\right)^{\frac{1}{2 \kappa}} \leq C_{1}^{C V}\left[(\Delta t)^{\frac{1}{2}}+\Delta \tau\right], \\
\max _{0 \leq n \leq N}\left(\mathbb{E}\left[\left|Y\left(t_{n}\right)-\overline{\bar{g}}_{n, L}\right|^{2 \kappa}\right]\right)^{\frac{1}{2 \kappa}} \leq C_{2}^{C V}\left[(\Delta t)^{\frac{1}{2}}+\Delta \tau\right], \\
\left(\mathbb{E}\left[\left|Z\left(t_{\max }\right)-\overline{\bar{g}}_{N, L+1}\right|^{2 \kappa}\right]\right)^{\frac{1}{2 \kappa}} \leq C_{3}^{C V}\left[(\Delta t)^{\frac{1}{2}}+\Delta \tau\right], \\
\left(\mathbb{E}\left[\left|\Lambda_{\Psi}(g)-\Lambda_{\Psi}(\overline{\bar{g}})\right|^{2 \kappa}\right]\right)^{\frac{1}{2 \kappa}} \leq C_{4}^{C V}\left[(\Delta t)^{\frac{1}{2}}+\Delta \tau\right], \\
\left(\mathbb{E}\left[|\mathcal{G}(g)-\mathcal{G}(\overline{\bar{g}})|^{2 \kappa}\right]\right)^{\frac{1}{2 \kappa}} \leq C_{5}^{C V}\left[(\Delta t)^{\frac{1}{2}}+\Delta \tau\right]
\end{gathered}
$$

and, for the (EFD) method,

$$
\max _{(n, \ell) \in \mathcal{M}}\left(\mathbb{E}\left[\left|\frac{g\left(t_{n}, \tau_{\ell+1}\right)-g\left(t_{n}, \tau_{\ell}\right)}{\Delta \tau_{\ell}}-\frac{\overline{\bar{g}}_{n, \ell+1}-\overline{\bar{g}}_{n, \ell}}{\Delta \tau_{\ell}}\right|^{2 \kappa}\right]\right)^{\frac{1}{2 \kappa}} \leq C_{6}^{C V}\left[(\Delta t)^{\frac{1}{2}}+\Delta \tau\right] .
$$

Proof. Here, we set $D_{\star}:=\left[0, t_{\max }\right] \times\left[0, \tau_{\max }\right]$ and will use the symbol $C$ for a generic constant independent of the partitions of the intervals $\left[0, t_{\max }\right]$ and $\left[0, \tau_{\max }\right]$. Let $E_{m, \ell}=\widehat{g}_{m, \ell}-\overline{\bar{g}}_{m, \ell}$ for $m=0, \ldots, N$ and $\ell=0, \ldots, L-1$, where $\widehat{g}_{m, \ell}=g\left(t_{m}, \tau_{\ell}\right)$ for the (EFD) method and $\widehat{g}_{m, \ell}=\Pi g\left(t_{m} ; \tau_{\ell}\right)$ for the (EFE) method. First, subtract (2.6) or (2.8) from (3.38), and then sum with respect to $n$, to obtain

$$
E_{n, \ell}=A_{n, \ell}+B_{n, \ell}+\sum_{m=0}^{n-1} \mathcal{K}_{m, \ell}, \quad \ell=0, \ldots, L-1, \quad n=1, \ldots, N,
$$

where

$$
\begin{aligned}
A_{n, \ell} & :=\sum_{m=0}^{n-1} \Delta t_{m} \nu^{m, \ell}\left[\xi^{2}\left(\widehat{g}_{m, \ell_{m}}+f_{0}^{m}\right)-\xi^{2}\left(\overline{\bar{g}}_{m, \ell_{m}}+f_{0}^{m}\right)\right], \\
B_{n, \ell} & :=\sum_{m=0}^{n-1} \sum_{j=1}^{J} \mu_{j}^{m, \ell}\left[\xi\left(\widehat{g}_{m, \ell_{m}}+f_{0}^{m}\right)-\xi\left(\overline{\bar{g}}_{m, \ell_{m}}+f_{0}^{m}\right)\right] \Delta W_{m}^{j},
\end{aligned}
$$


$f_{0}^{m}:=f_{0}\left(t_{m}\right), \nu^{m, \ell}=\widetilde{\lambda}\left(t_{m}, \tau_{\ell}\right)$ and $\mu_{j}^{m, \ell}=\lambda_{j}\left(t_{m}, \tau_{\ell}\right)$ for the (EFD) method and $\nu^{m, \ell}=\Pi \widetilde{\lambda}\left(t_{m} ; \tau_{\ell}\right)$ and $\mu_{j}^{m, \ell}=\Pi \lambda_{j}\left(t_{m} ; \tau_{\ell}\right)$ for the (EFE) method. Thus, we have

$$
\mathbb{E}\left[\left|E_{n, \ell}\right|^{2 \kappa}\right]=3^{2 \kappa-1}\left(\mathbb{E}\left[\left|A_{n, \ell}\right|^{2 \kappa}\right]+\mathbb{E}\left[\left|B_{n, \ell}\right|^{2 \kappa}\right]+\mathbb{E}\left[\left|\sum_{m=1}^{n-1} \mathcal{K}_{m, \ell}\right|^{2 \kappa}\right]\right)
$$

for $\ell=0, \ldots, L-1$ and $n=1, \ldots, N$. First, using (3.2) and the Hölder inequality, we obtain

$$
\begin{aligned}
\mathbb{E}\left[\left|A_{n, \ell}\right|^{2 \kappa}\right] & \leq\left(C_{\xi, 2}\right)^{2} \max _{D_{\star}}|\widetilde{\lambda}|^{2 \kappa} \mathbb{E}\left[\left(\sum_{m=0}^{n-1} \Delta t_{m}\left|E_{m, \ell_{m}}\right|\right)^{2 \kappa}\right] \\
& \leq C\left(\sum_{m=0}^{n-1} \Delta t_{m} \mathbb{E}\left[\left(E_{m, \ell_{m}}\right)^{2 \kappa}\right]\right)
\end{aligned}
$$

and

$$
\begin{aligned}
\mathbb{E}\left[\left|B_{n, \ell}\right|^{2 \kappa}\right] & \leq C \sum_{j=1}^{J}\left(\sum_{m=0}^{n-1} \Delta t_{m}\left(\mu_{j}^{m, \ell}\right)^{2} \mathbb{E}\left[\left|\xi\left(\widehat{g}_{m, \ell_{m}}+f_{0}^{m}\right)-\xi\left(\overline{\bar{g}}_{m, \ell_{m}}+f_{0}^{m}\right)\right|^{2}\right]\right)^{\kappa} \\
& \leq C\left(\sum_{m=0}^{n-1} \Delta t_{m} \mathbb{E}\left[\left|\xi\left(\widehat{g}_{m, \ell_{m}}+f_{0}^{m}\right)-\xi\left(\overline{\bar{g}}_{m, \ell_{m}}+f_{0}^{m}\right)\right|^{2}\right]\right)^{\kappa} \\
& \leq C\left(\sum_{m=0}^{n-1} \Delta t_{m} \mathbb{E}\left[\left(E_{m, \ell_{m}}\right)^{2}\right]\right)^{\kappa}
\end{aligned}
$$

for $\ell=0, \ldots, L-1$ and $n=1, \ldots, N$. Combining, (3.56), (3.57) and (3.58) and (3.39), we have

$$
\mathbb{E}\left[\left|E_{n, \ell}\right|^{2 \kappa}\right] \leq C\left[\left((\Delta t)^{\kappa}+(\Delta \tau)^{2 \kappa}\right)+\sum_{m=0}^{n-1} \Delta t_{m} \mathbb{E}\left[\left|E_{m, \ell_{m}}\right|^{2 \kappa}\right]+\left(\sum_{m=0}^{n-1} \Delta t_{m} \mathbb{E}\left[\left|E_{m, \ell_{m}}\right|^{2}\right]\right)^{\kappa}\right]
$$

for $\ell=0, \ldots, L-1$ and $n=1, \ldots, N$. Considering the case $\kappa=1$ and proceeding as in the proof of Lemma 3.3 from (3.59) we arrive at the estimate

$$
\max _{0 \leq n \leq N} \mathbb{E}\left[\left|E_{n, \ell_{n}}\right|^{2}\right] \leq C\left(\Delta t+(\Delta \tau)^{2}\right)
$$

Letting $\kappa \geq 2$, under the view of (3.60), the inequality (3.59) yields

$$
\mathbb{E}\left[\left|E_{n, \ell}\right|^{2 \kappa}\right] \leq C\left[\left((\Delta t)^{\kappa}+(\Delta \tau)^{2 \kappa}\right)+\sum_{m=0}^{n-1} \Delta t_{m} \mathbb{E}\left[\left|E_{m, \ell_{m}}\right|^{2 \kappa}\right]\right]
$$

for $\ell=0, \ldots, L-1$ and $n=1, \ldots, N$. Now, proceeding again as in the proof of Lemma 3.3 from (3.61) we conclude that

$$
\max _{0 \leq n \leq N} \mathbb{E}\left[\left|E_{n, \ell_{n}}\right|^{2 \kappa}\right] \leq C\left((\Delta t)^{\kappa}+(\Delta \tau)^{2 \kappa}\right) .
$$

Thus, combining (3.61) and (3.62) we arrive at

$$
\max _{(n, \ell) \in \mathcal{I}_{N, L}} \mathbb{E}\left[\left|E_{n, \ell}\right|^{2 \kappa}\right] \leq C\left((\Delta t)^{\kappa}+(\Delta \tau)^{2 \kappa}\right) .
$$

The estimate (3.50) for the (EFD) method follows directly from (3.63). For the (EFE) method, (3.50) follows combining (3.63) with the following estimate (cf. (3.47)

$$
\begin{aligned}
\max _{(n, \ell) \in \mathcal{I}_{N, L}} \mathbb{E}\left[\left|g\left(t_{n}, \tau_{\ell}\right)-\Pi g\left(t_{n} ; \tau_{\ell}\right)\right|^{2 \kappa}\right] & \leq \max _{(n, \ell) \in \mathcal{I}} \max _{\tau \in\left[\tau_{\ell}, \tau_{\ell+1}\right]} \mathbb{E}\left[\left|g\left(t_{m}, \tau\right)-g\left(t_{m}, \tau_{\ell}\right)\right|^{2 \kappa}\right] \\
& \leq C(\Delta \tau)^{2 \kappa} .
\end{aligned}
$$

Since $\frac{E_{n, \ell+1}-E_{n, \ell}}{\Delta \tau_{\ell}}=\frac{A_{n, \ell+1}-A_{n, \ell}}{\Delta \tau_{\ell}}+\frac{B_{n, \ell+1}-B_{n, \ell}}{\Delta \tau_{\ell}}+\sum_{m=0}^{n-1} \frac{\mathcal{K}_{m, \ell+1}-\mathcal{K}_{m, \ell}}{\Delta \tau_{\ell}}$ for $\ell=0, \ldots, L-2$ and $n=1, \ldots, N$, to obtain the estimate (3.55) for the (EFD) method we proceed as above using (3.40) and (3.49). 
In order to get the second error estimate, we use (1.6) and (2.6) or (2.8), to conclude that

$$
\mathbb{E}\left[\left|Y\left(t_{n}\right)-\overline{\bar{g}}_{n, L}\right|^{2 \kappa}\right] \leq C \sum_{i=1}^{3} \mathbb{E}\left[\left|\zeta_{i}^{n}\right|^{2 \kappa}\right], \quad n=1, \ldots, N,
$$

where

$$
\begin{gathered}
\zeta_{1}^{n}:=\sum_{m=0}^{n-1} \int_{t_{m}}^{t_{m+1}}\left(f_{0}(s)-f_{0}\left(t_{m}\right)\right) d s, \quad \zeta_{2}^{n}:=\sum_{m=0}^{n-1} \int_{t_{m}}^{t_{m+1}}\left(g(s, s)-g\left(t_{m}, \tau_{\ell_{m}}\right)\right) d s, \\
\zeta_{3}^{n}:=\sum_{m=0}^{n-1} \Delta t_{m}\left(g\left(t_{m}, \tau_{\ell_{m}}\right)-\overline{\bar{g}}_{m, \ell_{m}}\right) .
\end{gathered}
$$

First, we observe that

$$
\left|\zeta_{1}^{n}\right|^{2 \kappa} \leq C(\Delta t)^{2 \kappa} \max _{\left[0, t_{\max }\right]}\left|f_{0}^{\prime}\right|^{2 \kappa}, \quad n=1, \ldots, N .
$$

Next, we use the Hölder inequality, (3.34), 3.33) and (3.50) to obtain

$$
\begin{aligned}
\mathbb{E}\left[\left|\zeta_{2}^{n}\right|^{2 \kappa}\right] & \leq C \sum_{m=0}^{n-1} \int_{t_{m}}^{t_{m+1}} \mathbb{E}\left[\left|g(s, s)-g\left(t_{m}, \tau_{\ell_{m}}\right)\right|^{2 \kappa}\right] d s \\
& \leq C \sum_{m=0}^{n-1} \int_{t_{m}}^{t_{m+1}} \mathbb{E}\left[\left|g(s, s)-g\left(t_{m}, s\right)\right|^{2 \kappa}+\left|g\left(t_{m}, s\right)-g\left(t_{m}, \tau_{\ell_{m}}\right)\right|^{2 \kappa}\right] d s \\
& \leq C\left[(\Delta t)^{\kappa}+\sum_{m=0}^{n-1} \int_{t_{m}}^{t_{m+1}}\left(\left|s-t_{m}\right|^{2 \kappa}+\left|t_{m}-\tau_{\ell_{m}}\right|^{2 \kappa}\right) d s\right] \\
& \leq C\left[(\Delta t)^{\kappa}+\sum_{m=0}^{n-1} \Delta t_{m}\left|\tau_{\ell_{m}+1}-\tau_{\ell_{m}}\right|^{2 \kappa}\right] \\
& \leq C\left[(\Delta t)^{\kappa}+(\Delta \tau)^{2 \kappa}\right], \quad n=1, \ldots, N,
\end{aligned}
$$

and

$$
\begin{aligned}
\mathbb{E}\left[\left|\zeta_{3}^{n}\right|^{2 \kappa}\right] & \leq C \sum_{m=0}^{n-1} \Delta t_{m} \mathbb{E}\left[\left|g\left(t_{m}, \tau_{\ell_{m}}\right)-\overline{\bar{g}}_{m, \ell_{m}}\right|^{2 \kappa}\right] \\
& \leq C\left[(\Delta t)^{\kappa}+(\Delta \tau)^{2 \kappa}\right], \quad n=1, \ldots, N .
\end{aligned}
$$

Thus, (3.51) follows easily from (3.68), (3.65), (3.66) and (3.67).

In order to prove our third error estimate, we use (1.6), (2.6) or (2.8), and the mean value theorem for scalar fields, to conclude that

$$
\mathbb{E}\left[\left|Z\left(t_{\max }\right)-\overline{\bar{g}}_{N, L+1}\right|^{2 \kappa}\right] \leq C \sum_{i=1}^{3} \mathbb{E}\left[\left|\Gamma_{i}\right|^{2 \kappa}\right],
$$

where

$$
\begin{aligned}
\Gamma_{1} & :=\sum_{m=0}^{N-1} \int_{t_{m}}^{t_{m+1}} F^{\prime}\left(A_{m}(s)\right) U\left(B_{m}(s)\right)\left(Y(s)-\overline{\bar{g}}_{m, L}\right) d s, \\
\Gamma_{2} & :=\sum_{m=0}^{N-1} \int_{t_{m}}^{t_{m+1}} F\left(A_{m}(s)\right) U^{\prime}\left(B_{m}(s)\right)\left(g(s, s)-\overline{\bar{g}}_{m, \ell_{m}}\right) d s, \\
\Gamma_{3} & :=\sum_{m=0}^{N-1} \int_{t_{m}}^{t_{m+1}} F\left(A_{m}(s)\right) U^{\prime}\left(B_{m}(s)\right)\left(f_{0}(s)-f_{0}^{m}\right) d s,
\end{aligned}
$$

and

$$
\begin{aligned}
& A_{m}(s):=\delta_{m}(s)\left(Y(s)-\overline{\bar{g}}_{m, L}\right)+\overline{\bar{g}}_{m, L}, \\
& B_{m}(s):=\tilde{\delta}_{m}(s)\left(g(s, s)+f_{0}(s)\right)+\left(1-\tilde{\delta}_{m}(s)\right)\left(\overline{\bar{g}}_{m, \ell_{m}}+f_{0}^{m}\right),
\end{aligned}
$$


with $\delta_{m}(s), \tilde{\delta}_{m}(s) \in[0,1]$. Let $\tilde{m} \in \mathbb{N}$. Since $F, F^{\prime}, U$ and $U^{\prime}$ have polynomial growth, we use (3.51), (3.12), (3.20) and (3.3) to conclude that there exist a nonnegative constant $C_{\star}^{m}$ such that

$$
\max _{0 \leq m \leq N-1} \sup _{s \in\left(t_{m}, t_{m+1}\right)}\left[\mathbb{E}\left[\left|F^{\prime}\left(A_{m}(s)\right) U\left(B_{m}(s)\right)\right|^{2 \tilde{m}}\right]+\mathbb{E}\left[\left|F\left(A_{m}(s)\right) U^{\prime}\left(B_{m}(s)\right)\right|^{2 \tilde{m}}\right]\right] \leq C_{\star}^{\tilde{m}} .
$$

Also, we use the Hölder inequality and (3.3) to arrive at

$$
\begin{aligned}
\mathbb{E}\left[\left|Y\left(t_{b}\right)-Y\left(t_{a}\right)\right|^{2 \tilde{m}}\right] & \leq\left(t_{b}-t_{a}\right)^{2 \tilde{m}-1} \int_{t_{a}}^{t_{b}} \mathbb{E}\left[\left|g(s, s)+f_{0}(s)\right|^{2 \tilde{m}}\right] d s \\
& \leq C\left(t_{b}-t_{a}\right)^{2 \tilde{m}}
\end{aligned}
$$

for all $t_{a}, t_{b} \in\left[0, t_{\max }\right]$ with $t_{a} \leq t_{b}$. Now, we are ready to estimare the quantities at the right hand side of (3.68). First, we use the Hölder inequality and (3.69) to arrive at

$$
\begin{aligned}
& \mathbb{E}\left[\left|\Gamma_{1}\right|^{2 \kappa}\right] \leq C \sum_{m=0}^{N-1} \int_{t_{m}}^{t_{m+1}} \mathbb{E}\left[\left|F^{\prime}\left(A_{m}(s)\right) U\left(B_{m}(s)\right)\right|^{2 \kappa}\left|Y(s)-\overline{\bar{g}}_{m, L}\right|^{2 \kappa}\right] d s, \\
& \leq C \sum_{m=0}^{N-1} \int_{t_{m}}^{t_{m+1}}\left(\mathbb{E}\left[\left|F^{\prime}\left(A_{m}(s)\right) U\left(B_{m}(s)\right)\right|^{4 \kappa}\right]\right)^{\frac{1}{2}}\left(\mathbb{E}\left[\left|Y(s)-\overline{\bar{g}}_{m, L}\right|^{4 \kappa}\right]\right)^{\frac{1}{2}} d s, \\
& \leq C \sum_{m=0}^{N-1} \int_{t_{m}}^{t_{m+1}}\left(\mathbb{E}\left[\left|Y(s)-Y\left(t_{m}\right)\right|^{4 \kappa}+\left|Y\left(t_{m}\right)-\overline{\bar{g}}_{m, L}\right|^{4 \kappa}\right]\right)^{\frac{1}{2}} d s, \\
& \mathbb{E}\left[\left|\Gamma_{2}\right|^{2 \kappa}\right] \leq C \sum_{m=0}^{N-1} \int_{t_{m}}^{t_{m+1}}\left(\mathbb{E}\left[\left|F\left(A_{m}(s)\right) U^{\prime}\left(B_{m}(s)\right)\right|^{4 \kappa}\right]\right)^{\frac{1}{2}}\left(\mathbb{E}\left[\left|g(s, s)-\overline{\bar{g}}_{m, \ell_{m}}\right|^{4 \kappa}\right]\right)^{\frac{1}{2}} d s \\
& \leq C \sum_{m=0}^{N-1} \int_{t_{m}}^{t_{m+1}}\left(\mathbb{E}\left[\left|g(s, s)-g\left(t_{m}, \tau_{\ell_{m}}\right)\right|^{4 \kappa}+\left|g\left(t_{m}, \tau_{\ell_{m}}\right)-\overline{\bar{g}}_{m, \ell_{m}}\right|^{4 \kappa}\right]\right)^{\frac{1}{2}} d s,
\end{aligned}
$$

and

$$
\mathbb{E}\left[\left|\Gamma_{3}\right|^{2 \kappa}\right] \leq C \sum_{m=0}^{N-1} \int_{t_{m}}^{t_{m+1}}\left|f_{0}(s)-f_{0}^{m}\right|^{2 \kappa} d s .
$$

Next, we combining (3.72), (3.73), (3.48) and (3.50) we obtain

$$
\mathbb{E}\left[\left|\Gamma_{2}\right|^{2 \kappa}\right]+\mathbb{E}\left[\left|\Gamma_{3}\right|^{2 \kappa}\right] \leq C\left[(\Delta t)^{\kappa}+(\Delta \tau)^{2 \kappa}\right] .
$$

Finally, we combine (3.71), (3.70) and (3.51) to obtain

$$
\mathbb{E}\left[\left|\Gamma_{1}\right|^{2 \kappa}\right] \leq C\left[(\Delta t)^{\kappa}+(\Delta \tau)^{2 \kappa}\right] .
$$

Thus, the error estimate (3.52) is a simple consequence of (3.68), (3.74) and (3.75).

To derive our fourth error estimate, first we set $E:=\Lambda_{\Psi}(g)-\Lambda_{\Psi}(\overline{\bar{g}})$, and then we use the Hölder inequality to obtain

$$
\mathbb{E}\left[|E|^{2 \kappa}\right] \leq C \sqrt{E_{A}} \sqrt{E_{B}}
$$

where

$$
\begin{aligned}
& E_{A}:=\sum_{\ell=\ell_{a}}^{L-1} \int_{\tau_{\ell}}^{\tau_{\ell+1}} \mathbb{E}\left[\sup _{\epsilon \in[0,1]}\left|\Psi^{\prime}\left(f_{0}(\tau)+\epsilon g\left(t_{\max }, \tau\right)+(1-\epsilon) \overline{\bar{g}}_{N, \ell}\right)\right|^{4 \kappa}\right] d \tau, \\
& E_{B}:=\sum_{\ell=\ell_{a}}^{L-1} \int_{\tau_{\ell}}^{\tau_{\ell+1}} \mathbb{E}\left[\left|g\left(t_{\max }, \tau\right)-\overline{\bar{g}}_{N, \ell}\right|^{4 \kappa}\right] d \tau .
\end{aligned}
$$

Since $\Psi^{\prime}$ has polynomial growth, the use of (3.20) and (3.3) yields that

$$
E_{A} \leq C \text {. }
$$

Also, using (3.33) and (3.50) we obtain

$$
E_{B} \leq C\left[(\Delta t)^{2 \kappa}+(\Delta \tau)^{4 \kappa}\right] .
$$

Thus, the estimate (3.53) follows after combining (3.76), (3.77) and (3.78). 
To obtain our fifth error estimate, first we set $E_{\mathcal{G}}:=\mathcal{G}(g)-\mathcal{G}(\overline{\bar{g}})$ and use the error bound (3.52) to obtain

$$
\mathbb{E}\left[\left|E_{\mathcal{G}}\right|^{2 \kappa}\right] \leq C\left[\sqrt{\mathcal{G}_{A_{1}}} \sqrt{\mathcal{G}_{A_{2}}}+\sqrt{\mathcal{G}_{B_{1}}} \sqrt{\mathcal{G}_{B_{2}}}+(\Delta t)^{\kappa}+(\Delta \tau)^{2 \kappa}\right]
$$

where

$$
\begin{array}{cc}
\mathcal{G}_{A_{1}}:=\mathbb{E}\left[\left|G\left(\Lambda_{\Psi}(g)\right)\right|^{4 \kappa}\right], \quad \mathcal{G}_{A_{2}}:=\mathbb{E}\left[\left|F\left(Y\left(t_{\max }\right)\right)-F\left(\overline{\bar{g}}_{N, L}\right)\right|^{4 \kappa}\right] \\
\mathcal{G}_{B_{1}}:=\mathbb{E}\left[\left|F\left(\overline{\bar{g}}_{N, L}\right)\right|^{4 \kappa}\right], \quad \mathcal{G}_{B_{2}}:=\mathbb{E}\left[\left|G\left(\Lambda_{\Psi}(g)\right)-G\left(\Lambda_{\Psi}(\overline{\bar{g}})\right)\right|^{4 \kappa}\right] .
\end{array}
$$

Since $F$ and $G$ have polynomial growth, we combine (3.12) and (3.51) to get

$$
\mathcal{G}_{A_{1}}+\mathcal{G}_{B_{1}} \leq C .
$$

Since $F^{\prime}$ has polynomial growth, we use the mean value theorem, the Cauchy-Schwarz inequality, (3.12) and the error bound (3.51) to have

$$
\begin{aligned}
\mathcal{G}_{A_{2}} & \leq\left(\mathbb{E}\left[\max _{\epsilon \in[0,1]}\left|F^{\prime}\left(\epsilon Y\left(t_{\max }\right)+(1-\epsilon) \overline{\bar{g}}_{N, L}\right)\right|^{8 \kappa}\right]\right)^{\frac{1}{2}}\left(\mathbb{E}\left[\left|Y\left(t_{\max }\right)-\overline{\bar{g}}_{N, L}\right|^{8 \kappa}\right]\right)^{\frac{1}{2}} \\
& \leq C\left[(\Delta t)^{2 \kappa}+(\Delta \tau)^{4 \kappa}\right] .
\end{aligned}
$$

Similarly, since $G^{\prime}$ has polynomial growth, we use the mean value theorem, the Cauchy-Schwarz inequality, (3.12), and the error bound (3.53) to have

$$
\begin{aligned}
\mathcal{G}_{B_{2}} & \leq\left(\mathbb{E}\left[\max _{\epsilon \in[0,1]}\left|G^{\prime}\left(\epsilon \Lambda_{\Psi}(g)+(1-\epsilon) \Lambda_{\Psi}(\overline{\bar{g}})\right)\right|^{8 \kappa}\right]\right)^{\frac{1}{2}}\left(\mathbb{E}\left[\left|\Lambda_{\Psi}(g)-\Lambda_{\Psi}(\overline{\bar{g}})\right|^{8 \kappa}\right]\right)^{\frac{1}{2}} \\
& \leq C\left[(\Delta t)^{2 \kappa}+(\Delta \tau)^{4 \kappa}\right] .
\end{aligned}
$$

Thus, the error bound (3.54) is a simple consequence of (3.79), (3.80), (3.81) and (3.82).

Theorem 3.3. Let $\mathcal{I}:=\{0, \ldots, N\} \times\{0, \ldots, L+1\},\left(\overline{\bar{g}}_{n, \ell}\right)_{(n, \ell) \in \mathcal{I}}$ be the numerical approximations produced by the $(\mathrm{EFD})$ or the $(\mathrm{EFM})$ method, $\Lambda_{\Psi}(\overline{\bar{g}})$ be defined as in the Theorem 3.2 and $\bar{\Lambda}_{\Psi, Q}(\overline{\bar{g}})$ be the quantity defined by (2.9). We assume that the quadrature rule $Q$ used in (2.9) is of order $p_{Q}$, $\Psi \in C^{p_{Q}}(\mathbb{R} ; \mathbb{R})$ and $f_{0} \in C^{p_{Q}}\left(\left[0, \tau_{\max }\right] ; \mathbb{R}\right)$. Also, we assume that $\Psi$ and all its derivatives up to order $p_{Q}$, along with the functions $F$ and $G^{\prime}$, have polynomial growth. Then, for $\kappa \in \mathbb{N}$, there exist constants $C_{\kappa}^{Q, A}$ and $C_{\kappa}^{Q, B}$, independent of the partitions of the intervals $\left[0, t_{\max }\right]$ and $\left[0, \tau_{\max }\right]$, such that

$$
\left(\mathbb{E}\left[\left|\Lambda_{\Psi}(\overline{\bar{g}})-\bar{\Lambda}_{\Psi, Q}(\overline{\bar{g}})\right|^{2 \kappa}\right]\right)^{\frac{1}{2 \kappa}} \leq C_{\kappa}^{Q, A}(\Delta \tau)^{p_{Q}}
$$

and

$$
\left(\mathbb{E}\left[|\mathcal{G}(\overline{\bar{g}})-\overline{\mathcal{G}}(\overline{\bar{g}})|^{2 \kappa}\right]\right)^{\frac{1}{2 \kappa}} \leq C_{\kappa}^{Q, B}(\Delta \tau)^{p_{Q}}
$$

Proof. For $\ell=\ell_{a}, \ldots, L-1$, we set $v_{\ell}(s):=\Psi\left(\overline{\bar{g}}_{N, \ell}+f_{0}\left(\tau_{\ell}+s \Delta \tau_{\ell}\right)\right)$ for $s \in[0,1]$. Since the quadrature rule $Q$ has order $p_{Q}$, applying a standard argument from the error analysis for quadrature rules based on the Taylor formula (see, e.g., [2]), we obtain

$$
\mathbb{E}\left[\left|\Lambda_{\Psi}(\overline{\bar{g}})-\bar{\Lambda}_{\Psi, Q}(\overline{\bar{g}})\right|^{2 \kappa}\right] \leq C(\Delta \tau)^{2 \kappa p_{Q}} \mathbb{E}\left[\max _{\ell_{a} \leq \ell \leq L-1} \max _{[0,1]}\left|\partial_{s}^{p_{Q}} v_{\ell}\right|^{2 \kappa}\right] .
$$

Observing that $\left.\partial_{s}^{p_{Q}} v_{\ell}(s)=\sum_{j=0}^{p_{Q}}\left(\begin{array}{c}p_{Q} \\ j\end{array}\right) \Psi^{(j)}\left(\overline{\bar{g}}_{N, \ell}+f_{0}\left(\tau_{\ell}+s \Delta \tau_{\ell}\right)\right) f_{0}^{\left(p_{Q}-j\right)}\left(\tau_{\ell}+s \Delta \tau_{\ell}\right)\right)$, assuming that $\Psi^{(j)}$ has polynomial growth $p_{j}$ for $j=1, \ldots, p_{Q}$, and using (3.20), we obtain

$$
\begin{aligned}
\mathbb{E}\left[\max _{\ell_{a} \leq \ell \leq L-1} \sup _{[0,1]}\left|\partial_{s}^{p_{Q}} v_{\ell}\right|^{2 \kappa}\right] & \leq C \max _{\ell_{a} \leq \ell \leq L-1} \sum_{j=0}^{p_{Q}}\left(1+\left|\overline{\bar{g}}_{N, \ell}\right|^{2 \kappa p_{j}}\right) \\
& \leq C .
\end{aligned}
$$

Now, combine (3.85) and (3.86) to arrive at (3.83). 
Since $F$ and $G^{\prime}$ have polynomial growth, using the Cauchy-Schwarz inequality, the mean value theorem, (3.51), (3.12), (3.83) and (3.53), we obtain

$$
\begin{aligned}
\mathbb{E}\left[|\mathcal{G}(\overline{\bar{g}})-\overline{\mathcal{G}}(\overline{\bar{g}})|^{2 \kappa}\right] & \leq C\left(\mathbb{E}\left[\left|F\left(\overline{\bar{g}}_{N, L}\right)\right|^{4 \kappa}\right]\right)^{\frac{1}{2}}\left(\mathbb{E}\left[\left|G\left(\Lambda_{\Psi}(\overline{\bar{g}})\right)-G\left(\bar{\Lambda}_{\Psi, Q}(\overline{\bar{g}})\right)\right|^{4 \kappa}\right]\right)^{\frac{1}{2}} \\
& \leq C\left(\mathbb{E}\left[\sup _{\epsilon \in[0,1]}\left|G^{\prime}\left(\epsilon \Lambda_{\Psi}(\overline{\bar{g}})+(1-\epsilon) \bar{\Lambda}_{\Psi, Q}(\overline{\bar{g}})\right)\right|^{8 \kappa}\right]\right)^{\frac{1}{4}}\left(\mathbb{E}\left[\left|\Lambda_{\Psi}(\overline{\bar{g}})-\bar{\Lambda}_{\Psi, Q}(\overline{\bar{g}})\right|^{8 \kappa}\right]\right)^{\frac{1}{4}} \\
& \leq C(\Delta \tau)^{2 \kappa p_{Q}}
\end{aligned}
$$

which yields the estimate (3.84).

\section{Computable Weak Error Approximation}

In this section we present a computable approximation for the weak $t-$ and $\tau-$ discretization error $E_{D}$ defined in (2.14) for the (EFD) method. In Theorem 4.1 below we give an estimate of $E_{D}$ which, as the step size of both the time and maturity time partitions go to zero and the number of realizations goes to infinity, is asymptotically correct. On the other hand, the statistical error $E_{S}$ can be analyzed by the Central Limit Theorem or Berry-Esseen Theorem, a standard procedure in Monte Carlo methods (cf. Section 5). While, in Theorem 3.3 we have estimated the quadrature error $E_{Q}$, concluding that when the order $p_{Q}$ of the quadrature rule $Q$ we use in (2.9) is sufficiently large, the quadrature error, $E_{Q}$, is a higher order term in the expansion of the computational error.

To have an easier access to the results and the techniques of [21, we reformulate problem (1.9)-(1.10), letting the process $g=g(t, \tau)$ be the solution of the problem

$$
\begin{aligned}
d g(t, \tau) & =a(t, \tau, g(t, t)) d t+b(t, \tau, g(t, t)) \cdot d W(t), \quad \forall t \in\left[0, t_{\max }\right], \\
g(0, \tau) & =0
\end{aligned}
$$

for $\tau \in\left[0, \tau_{\max }\right]$, where $a:\left[0, t_{\max }\right] \times\left[0, \tau_{\max }\right] \times \mathbb{R} \rightarrow \mathbb{R}, b:\left[0, t_{\max }\right] \times\left[0, \tau_{\max }\right] \times \mathbb{R} \rightarrow \mathbb{R}^{J}$ given by

$$
\begin{aligned}
a(t, \tau, x) & \equiv \xi^{2}\left(x+f_{0}(t)\right) \tilde{\lambda}(t, \tau), \\
b(t, \tau, x) & \equiv \xi\left(x+f_{0}(t)\right) \lambda(t, \tau) .
\end{aligned}
$$

We approximate the unknown process $g(t, \tau)$ by a time and maturity discretization $\overline{\bar{g}}(t, \tau)$, with $t \in$ $\left(t_{n}\right)_{n=0}^{N}$ and $\tau \in\left(\tau_{\ell}\right)_{\ell=0}^{L-1}$, based on the (EFD) method, which, for $n=0, \ldots, N-1$, reads

$$
\begin{aligned}
\overline{\bar{g}}\left(t_{n+1}, \tau_{\ell}\right) & =\overline{\bar{g}}\left(t_{n}, \tau_{\ell}\right)+a\left(t_{n}, \tau_{\ell}, \overline{\bar{g}}\left(t_{n}, \tau_{\ell_{n}}\right)\right) \Delta t_{n}+b\left(t_{n}, \tau_{\ell}, \overline{\bar{g}}\left(t_{n}, \tau_{\ell_{n}}\right)\right) \cdot \Delta W_{n}, \quad \ell=0, \ldots, L-1, \\
\overline{\bar{g}}\left(0, \tau_{\ell}\right) & =0, \quad \ell=0, \ldots, L .
\end{aligned}
$$

For the analysis of the (EFD) method, it is useful to extend its definition for all times $t$ and all maturities $\tau$ as follows: for $n=0, \ldots, N-1$ and $\ell=0, \ldots, L-1$, set

$$
\begin{aligned}
\overline{\bar{g}}(t, \tau) & =\overline{\bar{g}}\left(t_{n}, \tau_{\ell}\right)+a\left(t_{n}, \tau_{\ell}, \overline{\bar{g}}\left(t_{n}, \tau_{\ell_{n}}\right)\right)\left(t-t_{n}\right)+b\left(t_{n}, \tau_{\ell}, \overline{\bar{g}}\left(t_{n}, \tau_{\ell_{n}}\right)\right) \cdot\left(W(t)-W\left(t_{n}\right)\right) \\
& =\overline{\bar{g}}\left(t_{n}, \tau_{\ell}\right)+\int_{t_{n}}^{t} \overline{\bar{a}}\left(s, \tau, \overline{\bar{g}}\left(t_{n}, \tau_{\ell_{n}}\right)\right) d s+\int_{t_{n}}^{t} \overline{\bar{b}}\left(s, \tau, \overline{\bar{g}}\left(t_{n}, \tau_{\ell_{n}}\right)\right) \cdot d W(s), \quad \forall t \in\left[t_{n}, t_{n+1}\right), \\
\overline{\bar{g}}(0, \tau) & =0,
\end{aligned}
$$

for $\tau \in\left[\tau_{\ell}, \tau_{\ell+1}\right)$, where $\overline{\bar{a}}$ and $\overline{\bar{b}}$ are the piecewise constant approximations

$$
\begin{aligned}
& \left.\overline{\bar{a}}(t, \tau, x)\right|_{(t, \tau) \in\left[t_{n}, t_{n+1}\right) \times\left[\tau_{\ell}, \tau_{\ell+1}\right)} \equiv a\left(t_{n}, \tau_{\ell}, x\right)=\xi^{2}\left(x+f_{0}\left(t_{n}\right)\right) \widetilde{\lambda}\left(t_{n}, \tau_{\ell}\right), \\
& \left.\overline{\bar{b}}(t, \tau, x)\right|_{(t, \tau) \in\left[t_{n}, t_{n+1}\right) \times\left[\tau_{\ell}, \tau_{\ell+1}\right)} \equiv b\left(t_{n}, \tau_{\ell}, x\right)=\xi\left(x+f_{0}\left(t_{n}\right)\right) \lambda\left(t_{n}, \tau_{\ell}\right) .
\end{aligned}
$$

Thus, the extension above results in $\overline{\bar{g}}(t,.) \in S_{\Delta \tau}$ for any time $t \in\left[0, t_{\max }\right]$.

Theorem 4.1. Let $\mathcal{I}:=\{0, \ldots, N\} \times\{0, \ldots, L-1\},\left(\overline{\bar{g}}_{n, \ell}\right)_{(n, \ell) \in \mathcal{I}}$ be the numerical approximations produced by the (EFD) method. Also, we assume that the functions $F, U, \Psi, G$ along with their derivatives have polynomial growth. Also, we set

$$
d(t, \tau, \widetilde{\tau}, x):=\frac{1}{2} \xi^{2}\left(x+f_{0}(t)\right) \lambda(t, \widetilde{\tau}) \cdot \lambda(t, \tau),
$$


for $x \in \mathbb{R}, t \in\left[0, t_{\max }\right]$ and $\tau, \widetilde{\tau} \in\left[0, \tau_{\max }\right]$. Then the computational error of the (EFD) method has the expansion

$$
E_{D}:=\mathbb{E}[\mathcal{G}(g)]-\mathbb{E}[\mathcal{G}(\overline{\bar{g}})]=E_{D, \mathrm{tau}}+E_{D, \mathrm{tim}}+\mathcal{O}\left((\Delta t)^{2}+(\Delta \tau)^{2}\right)
$$

where

$$
\begin{aligned}
E_{D, \mathrm{tau}}= & \sum_{n=0}^{N-1} \Delta t_{n}\left\{\sum_{\ell=0}^{L-1} \Delta \tau_{\ell} \mathbb{E}\left[\frac{a\left(t_{n}, \tau_{\ell+1}, \overline{\bar{g}}\left(t_{n}, \tau_{\ell_{n}}\right)\right)-a\left(t_{n}, \tau_{\ell}, \overline{\bar{g}}\left(t_{n}, \tau_{\ell_{n}}\right)\right)}{2} \overline{\bar{\varphi}}_{n, \ell}\right]\right\} \\
& +\sum_{n=0}^{N-1} \Delta t_{n}\left\{\sum_{\ell=0}^{L-1} \sum_{\ell^{\prime}=0}^{L-1} \Delta \tau_{\ell} \Delta \tau_{\ell^{\prime}} \mathbb{E}\left[\frac{d\left(t_{n}, \tau_{\ell+1}, \tau_{\ell^{\prime}+1}, \overline{\bar{g}}\left(t_{n}, \tau_{\ell_{n}}\right)\right)-d\left(t_{n}, \tau_{\ell}, \tau_{\ell^{\prime}}, \overline{\bar{g}}\left(t_{n}, \tau_{\ell_{n}}\right)\right)}{2} \overline{\bar{\varphi}}_{n, \ell, \ell^{\prime}}^{\prime}\right]\right\},
\end{aligned}
$$

and

$$
\begin{aligned}
E_{D, \text { tim }}=\sum_{n=0}^{N-1} \frac{\Delta t_{n}}{2}\left\{\mathbb{E}\left[\left(F\left(\overline{\bar{g}}_{n+1, L}\right) U\left(\overline{\bar{r}}_{n+1}\right)-F\left(\overline{\bar{g}}_{n, L}\right) U\left(\overline{\bar{r}}_{n}\right)\right) \overline{\bar{\varphi}}_{n+1, L+1}\right]\right. \\
+\mathbb{E}\left[\left(\overline{\bar{r}}_{n+1}-\overline{\bar{r}}_{n}\right) \overline{\bar{\varphi}}_{n+1, L}\right] \\
\left.+\sum_{\ell=0}^{L-1} \mathbb{E}\left[\left(a\left(t_{n+1}, \tau_{\ell}, \overline{\bar{g}}\left(t_{n+1}, t_{n+1}\right)\right)-a\left(t_{n}, \tau_{\ell}, \overline{\bar{g}}\left(t_{n}, t_{n}\right)\right)\right) \overline{\bar{\varphi}}_{n+1, \ell}\right]\right\} \\
+\sum_{n=0}^{N-1} \frac{\Delta t_{n}}{2}\left\{\sum_{\ell, \ell^{\prime}=0}^{L-1} \mathbb{E}\left[\left(d\left(t_{n+1}, \tau_{\ell}, \tau_{\ell^{\prime}}, \overline{\bar{g}}\left(t_{n+1}, t_{n+1}\right)\right)-d\left(t_{n}, \tau_{\ell}, \tau_{\ell^{\prime}}, \overline{\bar{g}}\left(t_{n}, t_{n}\right)\right)\right) \overline{\bar{\varphi}}_{n+1, \ell, \ell^{\prime}}^{\prime}\right]\right\}
\end{aligned}
$$

with

$$
\overline{\bar{r}}_{n}:=\overline{\bar{g}}\left(t_{n}, t_{n}\right)+f_{0}\left(t_{n}\right)=\overline{\bar{g}}_{n, \ell_{n}}+f_{0}\left(t_{n}\right) .
$$

The two leading order terms $E_{D, \text { tau }}$ and $E_{D, \text { tim }}$ in the right hand side of (4.6) are in a posteriori form and based on the discrete duals $\overline{\bar{\varphi}}_{n} \in \mathbb{R}^{L+2}$ and $\overline{\bar{\varphi}}_{n}^{\prime} \in \mathbb{R}^{(L+2) \times(L+2)}$ which are determined as follows. First, set

$$
\begin{aligned}
& \bar{\Lambda}_{\Psi, Q, \ell}^{\prime}(\overline{\bar{g}}):=\Delta \tau_{\ell} \sum_{i=1}^{N_{Q}} w_{Q, i} \Psi^{\prime}\left(\overline{\bar{g}}_{N, \ell}+f_{0}\left(\tau_{\ell}+s_{Q, i} \Delta \tau_{\ell}\right)\right), \\
& \bar{\Lambda}_{\Psi, Q, \ell}^{\prime \prime}(\overline{\bar{g}}):=\Delta \tau_{\ell} \sum_{i=1}^{N_{Q}} w_{Q, i} \Psi^{\prime \prime}\left(\overline{\bar{g}}_{N, \ell}+f_{0}\left(\tau_{\ell}+s_{Q, i} \Delta \tau_{\ell}\right)\right)
\end{aligned}
$$

for $\ell=\ell_{a}, \ldots, L-1$, and

$$
c_{n, j}(x):=a\left(t_{n}, \tau_{j}, x\right) \Delta t_{n}+b\left(t_{n}, \tau_{j}, x\right) \cdot \Delta W_{n}
$$

for $x \in \mathbb{R}$ and $j=0, \ldots, L-1$. Then, the first dual $\overline{\bar{\varphi}}$ is defined by the dual backward problem with final datum

$$
\overline{\bar{\varphi}}_{N, \ell}= \begin{cases}0, & \ell=0, \ldots, \ell_{a}-1, \\ F\left(\overline{\bar{g}}_{N, L}\right) G^{\prime}\left(\bar{\Lambda}_{\Psi, Q}(\overline{\bar{g}})\right) \bar{\Lambda}_{\Psi, Q, \ell}^{\prime}(\overline{\bar{g}}), & \ell=\ell_{a}, \ldots, L-1, \\ F^{\prime}\left(\overline{\bar{g}}_{N, L}\right) G\left(\bar{\Lambda}_{\Psi, Q}(\overline{\bar{g}})\right), & \ell=L, \\ 1, & \ell=L+1,\end{cases}
$$

and

$$
\overline{\bar{\varphi}}_{n, \ell}= \begin{cases}\overline{\bar{\varphi}}_{n+1, \ell}, & \ell \in\{0, \ldots, L-1\} \backslash\left\{\ell_{n}\right\}, \\ \Delta t_{n} \overline{\bar{\varphi}}_{n+1, L}+\Delta t_{n} F\left(\overline{\bar{g}}_{n, L}\right) U^{\prime}\left(\overline{\bar{r}}_{n}\right) \overline{\bar{\varphi}}_{n+1, L+1} & \\ \quad+\sum_{j=0}^{L-1} c_{n, j}^{\prime}\left(\overline{\bar{g}}_{n, \ell_{n}}\right) \overline{\bar{\varphi}}_{n+1, j}+\overline{\bar{\varphi}}_{n+1, \ell_{n}}, & \ell=\ell_{n} \\ \overline{\bar{\varphi}}_{n+1, L}+\Delta t_{n} F^{\prime}\left(\overline{\bar{g}}_{n, L}\right) U\left(\overline{\bar{r}}_{n}\right) \overline{\bar{\varphi}}_{n+1, L+1}, & \ell=L, \\ \overline{\bar{\varphi}}_{n+1, L+1}, & \ell=L+1,\end{cases}
$$


for $n=N-1, \ldots, 0$. The second dual, $\overline{\bar{\varphi}}^{\prime}$, has final datum

$$
(4.11) \quad \overline{\bar{\varphi}}_{N, \ell, \ell^{\prime}}^{\prime}= \begin{cases}0, & \ell=0, \ldots, \ell_{a}-1, \ell^{\prime}=0, \ldots, L+1, \\ F\left(\overline{\bar{g}}_{N, L}\right) G^{\prime \prime}\left(\bar{\Lambda}_{\Psi, Q}(\overline{\bar{g}})\right) \bar{\Lambda}_{\Psi, Q, \ell}(\overline{\bar{g}}) \bar{\Lambda}_{\Psi, Q, \ell^{\prime}}(\overline{\bar{g}}), & \ell, \ell^{\prime} \in\left\{\ell_{a}, \ldots, L-1\right\}, \ell \neq \ell^{\prime}, \\ F\left(\overline{\bar{g}}_{N, L}\right)\left[G^{\prime \prime}\left(\bar{\Lambda}_{\Psi, Q}(\overline{\bar{g}})\right)\left(\bar{\Lambda}_{\Psi, Q, \ell}^{\prime}(\overline{\bar{g}})\right)^{2}\right. & \\ \left.\quad+G^{\prime}\left(\bar{\Lambda}_{\Psi, Q}(\overline{\bar{g}})\right) \bar{\Lambda}_{\Psi, Q, \ell}^{\prime \prime}(\overline{\bar{g}})\right], & \ell \in\left\{\ell_{a}, \ldots, L-1\right\}, \ell^{\prime}=\ell, \\ F^{\prime}\left(\overline{\bar{g}}_{N, L}\right) G^{\prime}\left(\bar{\Lambda}_{\Psi, Q}(\overline{\bar{g}})\right) \bar{\Lambda}_{\Psi, Q, \ell}^{\prime}(\overline{\bar{g}}), & \ell=\ell_{a}, \ldots, L-1, \ell^{\prime}=L, \\ 0, & \ell=\ell_{a}, \ldots, L, \ell^{\prime}=L+1, \\ 0, & \ell=\ell_{a}, \ldots, L+1, \ell^{\prime}=0, \ldots, \ell_{a}-1, \\ F^{\prime}\left(\overline{\bar{g}}_{N, L}\right) G^{\prime}\left(\bar{\Lambda}_{\Psi, Q}(\overline{\bar{g}})\right) \bar{\Lambda}_{\Psi, Q, \ell^{\prime}}^{\prime}(\overline{\bar{g}}), & \quad \ell=L, \ell^{\prime}=\ell_{a}, \ldots, L-1, \\ F^{\prime \prime}\left(\overline{\bar{g}}_{N, L}\right) G\left(\bar{\Lambda}_{\Psi, Q}(\overline{\bar{g}})\right), & \ell=L, \ell^{\prime}=L, \\ 0, & \ell=L+1, \ell^{\prime}=\ell_{a}, \ldots, L+1,\end{cases}
$$

and solves the recursion

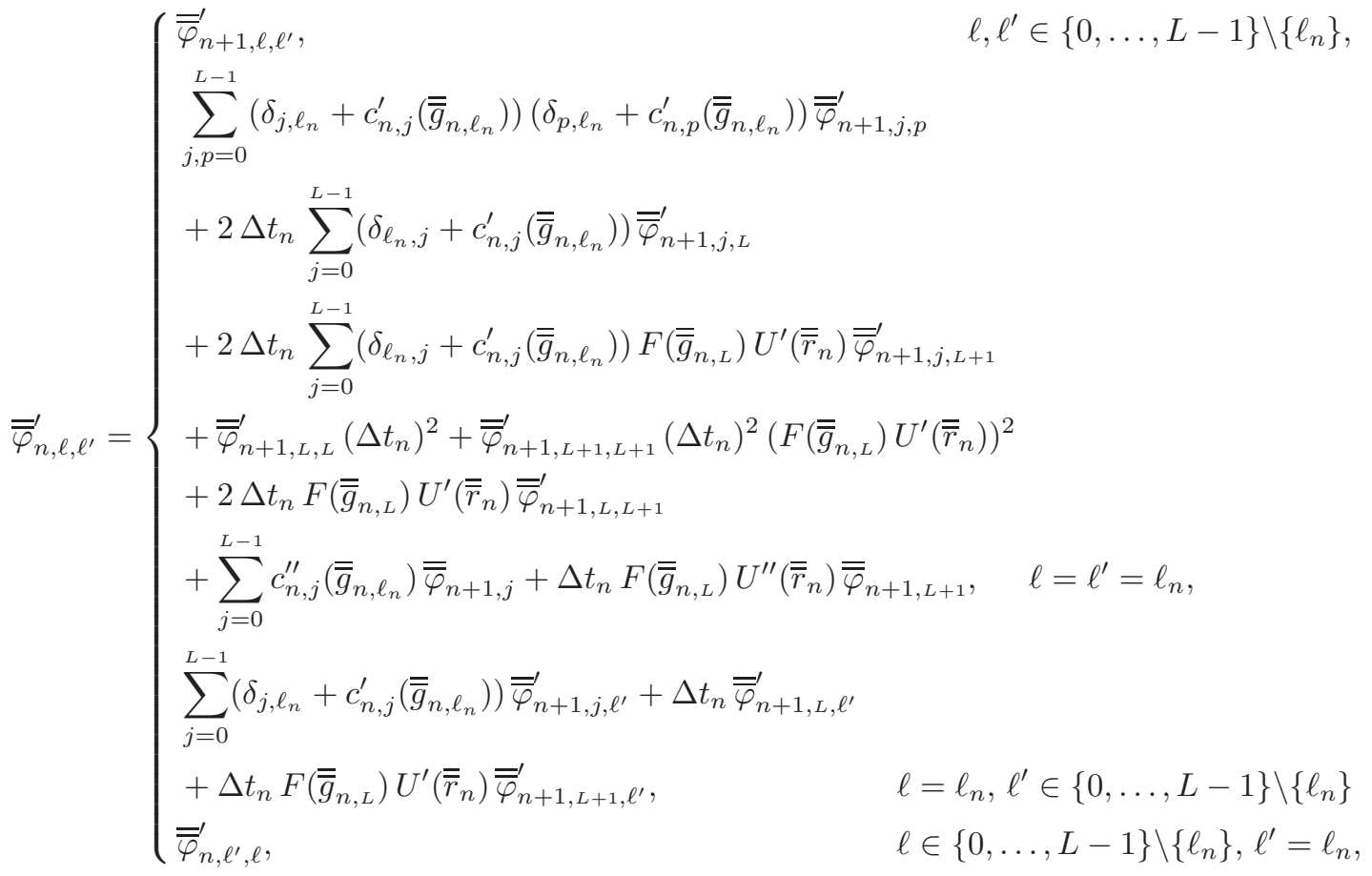

$$
\begin{aligned}
& \overline{\bar{\varphi}}_{n, \ell, \ell^{\prime}}^{\prime}= \begin{cases}\overline{\bar{\varphi}}_{n+1, L, \ell^{\prime}}^{\prime}+\Delta t_{n} F^{\prime}\left(\overline{\bar{g}}_{n, L}\right) U\left(\overline{\bar{r}}_{n}\right) \overline{\bar{\varphi}}_{n+1, L+1, \ell^{\prime}}^{\prime}, & \ell=L, \ell^{\prime} \in\{0, \ldots, L-1\} \backslash\left\{\ell_{n}\right\}, \\
\overline{\bar{\varphi}}_{n, \ell^{\prime}, \ell}^{\prime}, & \ell \in\{0, \ldots, L-1\} \backslash\left\{\ell_{n}\right\}, \ell^{\prime}=L,\end{cases} \\
& \overline{\bar{\varphi}}_{n, \ell, \ell^{\prime}}^{\prime}=\Delta t_{n} F^{\prime}\left(\overline{\bar{g}}_{n, L}\right) U^{\prime}\left(\overline{\bar{r}}_{n}\right) \overline{\bar{\varphi}}_{n+1, L+1}+\sum_{j=0}^{L-1}\left(\delta_{j, \ell_{n}}+c_{n, j}^{\prime}\left(\overline{\bar{g}}_{n, \ell_{n}}\right)\right) \overline{\bar{\varphi}}_{n+1, L, j}^{\prime} \\
& \left.+\Delta t_{n} F^{\prime}\left(\overline{\bar{g}}_{n, L}\right) U\left(\overline{\bar{r}}_{n}\right)\right) \sum_{j=0}^{L-1}\left(\delta_{j, \ell_{n}}+c_{n, j}^{\prime}\left(\overline{\bar{g}}_{n, \ell_{n}}\right)\right) \overline{\bar{\varphi}}_{n+1, L+1, j}^{\prime} \\
& \left.+\left(\Delta t_{n}\right)^{2} F^{\prime}\left(\overline{\bar{g}}_{n, L}\right) U\left(\overline{\bar{r}}_{n}\right)\right)\left[\overline{\bar{\varphi}}_{n+1, L+1, L}^{\prime}+F\left(\overline{\bar{g}}_{n, L}\right) U^{\prime}\left(\overline{\bar{r}}_{n}\right) \overline{\bar{\varphi}}_{n+1, L+1, L+1}^{\prime}\right] \\
& +\Delta t_{n}\left[\overline{\bar{\varphi}}_{n+1, L, L}^{\prime}+F\left(\overline{\bar{g}}_{n, L}\right) U^{\prime}\left(\overline{\bar{r}}_{n}\right) \overline{\bar{\varphi}}_{n+1, L, L+1}^{\prime}\right], \quad\left(\ell, \ell^{\prime}\right) \in\left\{\left(L, \ell_{n}\right),\left(\ell_{n}, L\right)\right\},
\end{aligned}
$$




$$
\begin{gathered}
\overline{\bar{\varphi}}_{n, \ell, \ell^{\prime}}^{\prime}=\Delta t_{n} F^{\prime \prime}\left(\overline{\bar{g}}_{n, L}\right) U\left(\overline{\bar{r}}_{n}\right) \overline{\bar{\varphi}}_{n+1, L+1}+\overline{\bar{\varphi}}_{n+1, L, L}^{\prime}+2 \Delta t_{n} F^{\prime}\left(\overline{\bar{g}}_{n, L}\right) U\left(\overline{\bar{r}}_{n}\right) \overline{\bar{\varphi}}_{n+1, L+1, L}^{\prime} \\
+\left(\Delta t_{n}\right)^{2}\left(F^{\prime}\left(\overline{\bar{g}}_{n, L}\right) U\left(\overline{\bar{r}}_{n}\right)\right)^{2} \overline{\bar{\varphi}}_{n+1, L+1, L+1}^{\prime}, \quad \ell=\ell^{\prime}=L, \\
\overline{\bar{\varphi}}_{n, \ell, \ell^{\prime}}^{\prime}=\overline{\bar{\varphi}}_{n+1, L, L+1}^{\prime}+\Delta t_{n} F^{\prime}\left(\overline{\bar{g}}_{n, L}\right) U\left(\overline{\bar{r}}_{n}\right) \overline{\bar{\varphi}}_{n+1, L+1, L+1}^{\prime}, \quad\left(\ell, \ell^{\prime}\right) \in\{(L, L+1),(L+1, L)\},
\end{gathered}
$$

and

$$
\overline{\bar{\varphi}}_{n, \ell, \ell^{\prime}}^{\prime}=\left\{\begin{array}{l}
\overline{\bar{\varphi}}_{n+1, L+1, \ell^{\prime}}^{\prime}, \quad \ell=L+1, \ell^{\prime} \in\{0, \ldots, L-1, L+1\} \backslash\left\{\ell_{n}\right\}, \\
\sum_{j=0}^{L-1}\left(\delta_{j, \ell_{n}}+c_{n, j}^{\prime}\left(\overline{\bar{g}}_{n, \ell_{n}}\right)\right) \overline{\bar{\varphi}}_{n+1, L+1, j}^{\prime}+\Delta t_{n} \overline{\bar{\varphi}}_{n+1, L+1, L}^{\prime} \\
+\Delta t_{n} F\left(\overline{\bar{g}}_{n, L}\right) U^{\prime}\left(\overline{\bar{r}}_{n}\right) \overline{\bar{\varphi}}_{n+1, L+1, L+1}^{\prime}, \quad \ell=L+1, \ell^{\prime}=\ell_{n}, \\
\overline{\bar{\varphi}}_{n, \ell^{\prime}, \ell}^{\prime}, \quad \ell \in\{0, \ldots, L-1, L+1\}, \ell^{\prime}=L+1 .
\end{array}\right.
$$

Proof. The proof is an application of Theorem 2.2 in [21. To be able to split the time and maturity time discretization errors, introduce the semidiscretized fluxes $\bar{a}$ and $\bar{b}$ that, for $\tau_{\ell} \leq \tau<\tau_{\ell+1}$, are defined as $\bar{a}(t, \tau, x) \equiv \xi^{2}\left(x+f_{0}(t)\right) \widetilde{\lambda}\left(t, \tau_{\ell}\right), \bar{b}(t, \tau, x) \equiv \xi\left(x+f_{0}(t)\right) \lambda\left(t, \tau_{\ell}\right)$ and denote by $\bar{g}$ the corresponding semidiscrete in $\tau$ solution. As a first step, replace the exact solution of (4.1), $g$, by a finite dimensional approximation: a piecewise constant $g_{*}(t, \cdot)$, which is an Euler approximation with a much finer discretization, both in time $t$ and maturity time $\tau$, than $\overline{\bar{g}}$. Thus, $g_{*}$ uses a time grid $\left(\hat{t}_{n}\right)_{n=0}^{P}$ much finer than $\left(t_{n}\right)_{n=0}^{N}$, and a maturity time grid, $\left(\hat{\tau}_{\ell}\right)_{\ell=0}^{M}$ much finer than $\left(\tau_{\ell}\right)_{\ell=0}^{L}$. Consequently, the number of time steps satisfy $P \gg N, M \gg L$, respectively, and $\Delta \hat{t}:=\max _{0 \leq m \leq P-1} \hat{t}_{m+1}-\hat{t}_{m} \ll \Delta t$, $\Delta \hat{\tau}:=\max _{0 \leq m \leq M-1} \hat{\tau}_{m+1}-\hat{\tau}_{m} \ll \Delta \tau$. In the application of Theorem 2.2 in [21], include the $\tau$ discretization error terms $a-\bar{a}, b-\bar{b}$ as well as the $t$-discretization terms $\bar{a}-\overline{\bar{a}}, \bar{b}-\overline{\bar{b}}$ in the error expansion, following Lemmata 2.1-2.5 in [21, to obtain (4.6-4.8) for $g$ replaced by the piecewise constant process $g_{*}$. For this purpose, observe that $\bar{g}$ can be also thought of as a piecewise constant function on the finer $\tau$-partition that defines $g_{*}$. The second step is to let $M, P \rightarrow \infty$ and $\Delta \hat{\tau}, \Delta \hat{t} \rightarrow 0$, using

$$
\left(\mathbb{E}\left[\max _{\left[0, \tau_{\max }\right]}\left|g(t, \cdot)-g_{*}(t, \cdot)\right|^{2}+\left|\frac{\left[g\left(t, \hat{\tau}_{m+1}\right)-g\left(t, \hat{\tau}_{m}\right)\right]-\left[g_{*}\left(t, \hat{\tau}_{m+1}\right)-g_{*}\left(t, \hat{\tau}_{m}\right)\right]}{\hat{\tau}_{m+1}-\hat{\tau}_{m}}\right|\right]\right)^{\frac{1}{2}}=\mathcal{O}\left(\Delta \hat{\tau}+(\Delta \hat{t})^{\frac{1}{2}}\right),
$$

for $t \in\left[0, t_{\max }\right]$ and $m=0, \ldots, M-2$, along with similar estimates for the corresponding dual functions $\overline{\bar{\varphi}}, \overline{\bar{\varphi}}^{\prime}, \ldots$, to control the higher order terms in the error expansion. The latter strong convergence estimates follow moving along the lines of the analysis of Section 3

Remark 4.1. In the (EFD) method the $\tau$-discretization error of (4.6) and (4.7) can, by (4.4), (4.5), be expressed by

$$
\begin{aligned}
E_{D, \text { tau }}= & \sum_{n=0}^{N-1} \Delta t_{n}\left\{\sum_{\ell=0}^{L-1} \Delta \tau_{\ell} \mathbb{E}\left[\xi^{2}\left(\overline{\bar{r}}_{n}\right) \overline{\bar{\varphi}}_{n, \ell}\right] \frac{\tilde{\lambda}\left(t_{n}, \tau_{\ell+1}\right)-\tilde{\lambda}\left(t_{n}, \tau_{\ell}\right)}{2}\right. \\
& \left.+\frac{1}{4} \sum_{\ell, \ell^{\prime}=0}^{L-1} \mathbb{E}\left[\xi^{2}\left(r_{n}\right) \overline{\bar{\varphi}}_{n, \ell, \ell^{\prime}}^{\prime}\right]\left[\lambda\left(t_{n}, \tau_{\ell+1}\right) \cdot \lambda\left(t_{n}, \tau_{\ell^{\prime}+1}\right)-\lambda\left(t_{n}, \tau_{\ell}\right) \cdot \lambda\left(t_{n}, \tau_{\ell^{\prime}}\right)\right] \Delta \tau_{\ell} \Delta \tau_{\ell^{\prime}}\right\}
\end{aligned}
$$

and the time discretization is

$$
\begin{aligned}
E_{D, \mathrm{tim}}=\sum_{n=0}^{N-1} \frac{\Delta t_{n}}{2}\left\{\mathbb{E}\left[F\left(\overline{\bar{g}}_{n+1, L}\right) U\left(\overline{\bar{r}}_{n+1}\right)-F\left(\overline{\bar{g}}_{n, L}\right) U\left(\overline{\bar{r}}_{n}\right)\right]+\mathbb{E}\left[\left(\overline{\bar{r}}_{n+1}-\overline{\bar{r}}_{n}\right) \overline{\bar{\varphi}}_{n+1, L}\right]\right. \\
+\sum_{\ell=0}^{L-1} \mathbb{E}\left[\left(\xi^{2}\left(\overline{\bar{r}}_{n+1}\right) \widetilde{\lambda}\left(t_{n+1}, \tau_{\ell}\right)-\xi^{2}\left(\overline{\bar{r}}_{n}\right) \widetilde{\lambda}\left(t_{n}, \tau_{\ell}\right)\right) \overline{\bar{\varphi}}_{n+1, \ell}\right] \\
+\frac{1}{2} \sum_{\ell, \ell^{\prime}=0}^{L-1} \mathbb{E}\left[\left(\xi^{2}\left(\overline{\bar{r}}_{n+1}\right) \lambda\left(t_{n+1}, \tau_{\ell}\right) \cdot \lambda\left(t_{n+1}, \tau_{\ell^{\prime}}\right)\right.\right. \\
\left.\left.\left.-\xi^{2}\left(\overline{\bar{r}}_{n}\right) \lambda\left(t_{n}, \tau_{\ell}\right) \cdot \lambda\left(t_{n}, \tau_{\ell^{\prime}}\right)\right) \overline{\bar{\varphi}}_{n+1, \ell, \ell^{\prime}}^{\prime}\right]\right\}
\end{aligned}
$$


In Monte Carlo computations all the expected values in (4.18) and (4.19) are naturally approximated by sample averages.

Remark 4.2. The analysis of the (EFE) method follows a similar line as the estimates of the (EFD) method. The difference lies in the $\tau$-discretization error, which by virtue of the orthogonality of both $\widetilde{\lambda}-\Pi \tilde{\lambda}$ and $\lambda-\Pi \lambda$ to the subspace of piecewise constant functions $S_{\Delta \tau}$, becomes second order accurate. Therefore, more careful expansions, including interpolation estimates, need to be carried out in order to capture the second order contributions from the $\tau$-discretization.

\section{NUMERICAL EXPERIMENTS}

In this section we provide numerical evidence for the weak computational error (2.12) of the numerical methods defined in Section 2 approximating the quantity of interest $\mathbb{E}[\mathcal{F}(f)]=\mathbb{E}[\mathcal{G}(g)]$ described in (1.4 1.6). In particular, we show results from numerical experiments with examples that have known exact solution which permit a straightforward derivation of an exact solution to compare with. The implementation uses double precision FORTRAN 77 and simulates the increments of the $J$ independent Wiener processes by a double precision modification of the functions ran 1 and gasdev proposed in 18 . The numerical quadrature approximation $\bar{\Lambda}_{\Psi, Q}(\overline{\bar{g}})$ of $\Lambda_{\Psi}(\overline{\bar{g}})$ in (2.9) is done via the use of Simpson's quadrature rule. For the particular case of the (EFD) method, the estimates for the computational error developed in Theorem 4.1 are compared with the exact computational error. The numerical results obtained are in agreement with the theory and the work to compute these estimates is small.

5.1. Control of the statistical error. For $M$ independent samples $\left\{Y\left(\omega_{j}\right)\right\}_{j=1}^{M}$ of a random variable $Y$, with $\mathbb{E}\left[|Y|^{6}\right]<\infty$, define the sample average $\mathcal{A}(Y ; M)$ and the sample standard deviation $\mathcal{S}(Y ; M)$ of $Y$ by

$$
\mathcal{A}(Y ; M) \equiv \frac{1}{M} \sum_{j=1}^{M} Y\left(\omega_{j}\right) \quad \text { and } \quad \mathcal{S}(Y ; M) \equiv\left[\mathcal{A}\left(Y^{2} ; M\right)-(\mathcal{A}(Y ; M))^{2}\right]^{\frac{1}{2}} .
$$

Let $\sigma \equiv \sqrt{\mathbb{E}\left[|Y-\mathbb{E}[Y]|^{2}\right]}$ and consider the random variable

$$
Z_{M} \equiv \frac{\sqrt{M}}{\sigma}(\mathcal{A}(Y ; M)-\mathbb{E}[Y])
$$

with cumulative distribution function $F_{Z_{M}}(x) \equiv P\left(Z_{M} \leq x\right)$, for $x \in \mathbb{R}$. Let

$$
\lambda \equiv \frac{1}{\sigma}\left(\mathbb{E}\left[|Y-\mathbb{E}[Y]|^{3}\right]\right)^{\frac{1}{3}}<\infty,
$$

then the Berry-Esseen theorem (cf. [10] p. 126), gives the following estimate in the central limit theorem

$$
\sup _{x \in \mathbb{R}}\left|F_{Z_{M}}(x)-\Phi(x)\right| \leq \frac{3}{\sqrt{M}} \lambda^{3}
$$

for the rate of convergence of $F_{Z_{M}}$ to the distribution function, $\Phi$, of a normal random variable with mean zero and variance one, i.e.

$$
\Phi(x)=\frac{1}{\sqrt{2 \pi}} \int_{-\infty}^{x} e^{-\frac{s^{2}}{2}} d s .
$$

Since in the examples below $M$ is sufficiently large, i.e. $M \gg 36 \lambda^{6}$, the statistical error

$$
\mathcal{E}_{S}(Y ; M) \equiv \mathbb{E}[Y]-\mathcal{A}(Y ; M)
$$

satisfies, by the Berry-Esseen theorem, the following probability approximation

$$
P\left(\left[\left|\mathcal{E}_{S}(Y ; M)\right| \leq c_{0} \frac{\sigma}{\sqrt{M}}\right]\right) \simeq 2 \Phi\left(c_{0}\right)-1 .
$$

In practice choose some constant $c_{0} \geq 1.65$, so the normal distribution satisfies

$$
1>2 \Phi\left(c_{0}\right)-1 \geq 0.901
$$

and the event

$$
\left|\mathcal{E}_{S}(Y ; M)\right| \leq \mathrm{E}_{S}(Y ; M) \equiv c_{0} \frac{\mathcal{S}(Y ; M)}{\sqrt{M}}
$$

has probability close to one, which involves the additional step to approximate $\sigma$ by $\mathcal{S}(Y ; M)$, cf. [11. Thus, in the computations $\mathrm{E}_{S}(Y ; M)$ is a good approximation of the statistical error $\mathcal{E}_{S}(Y ; M)$. 
For a given TOL $>0$, the goal is to find $M$ such that $\mathrm{E}_{S}(Y ; M) \leq$ TOL. The algorithm described in [21 adaptively finds the number of realizations $M$ to compute the sample average $\mathcal{A}(Y ; M)$ as an approximation to $\mathbb{E}[Y]$. With large probability, depending on $c_{0}$, the statistical error in the approximation is then bounded by TOL. For more details on the implementation of an adaptive algorithm to control the statistical error, see 21].

Remark 5.1 (Computational cost of the error estimates). The work to approximate $\mathbb{E}[\mathcal{G}(g)]=\mathbb{E}[X]$ within an accuracy TOL is $\mathcal{O}\left(\frac{\operatorname{Var}[X]}{\operatorname{TOL}^{4}}\right)$, provided we use the Monte Carlo version of the EFD method as in (2.11). It is therefore important to try to use both variance reduction techniques and adaptive methods to save computational effort. On the other hand, the work needed to compute sufficiently sharp error estimates as described in Theorem 4.1 is only $\mathcal{O}\left(\mathrm{TOL}^{-3}\right)$. The number of realizations needed to have a statistical error in the error bound much smaller than $\mathrm{TOL}$ is only $\mathcal{O}\left(\mathrm{TOL}^{-1}\right)$ instead of the $\mathcal{O}\left(\frac{\operatorname{Var}[X]}{\mathrm{TOL}^{2}}\right)$ realizations we need to compute an approximation of $\mathcal{F}(g)$ using (2.11), while the work to compute the error estimate for each realization is still $\mathcal{O}\left(\mathrm{TOL}^{-2}\right)$, including the computation of the duals $\overline{\bar{\varphi}}$ and $\overline{\bar{\varphi}}^{\prime}$. This surprising reduction of work for $\overline{\bar{\varphi}}$ and $\overline{\bar{\varphi}}^{\prime}$ is special for the HJM model studied here. For general SDEs the corresponding work would be $\mathcal{O}\left(\mathrm{TOL}^{-4}\right)$ instead of $\mathcal{O}\left(\mathrm{TOL}^{-2}\right)$. Thus, cheap and sharp error bounds are obtained by the use of the a posteriori error estimates in Theorem4.1. Observe that if variance reduction techniques are applied to the approximation of $\mathbb{E}[\mathcal{G}(g)]$, it is natural to try to use them also to reduce the variance in the error estimators.

Remark 5.2 (Variance reduction techniques). The use of variance reduction techniques can decrease substantially the statistical errors. In particular the so called antithetic variates technique introduced in [12] reduces the variance in a sample estimator $\mathcal{A}(M ; Y)$ by using another estimator $\mathcal{A}(M ; \widetilde{Y})$ with the same expectation as the first one, but which is negatively correlated with the first. Then, the improved estimator is $\mathcal{A}\left(M ; \frac{Y+\widetilde{Y}}{2}\right)$. Here, the choice of $Y$ and $\widetilde{Y}$ relates to the Wiener process $W$ and its reflection along the time axis, $-W$, which is also a Wiener process. If a realization of the Wiener process, $W\left(\cdot, \omega_{j}\right)$, yields, using one of the numerical discretizations (2.5]-2.8), a realization $\overline{\bar{g}}\left(\cdot, \cdot, \omega_{j}\right)$ and $-W\left(\cdot, \omega_{j}\right)$ yields $\overline{\overline{\widetilde{g}}}\left(\cdot, \cdot, \omega_{j}\right)$ respectively, then we choose

$$
\frac{1}{M} \sum_{j=1}^{M} \frac{F\left(\overline{\bar{g}}_{N, L}\left(\omega_{j}\right)\right) G\left(\bar{\Lambda}_{\Psi, Q}\left(\overline{\bar{g}}\left(\cdot, \cdot, \omega_{j}\right)\right)\right)+\overline{\bar{g}}_{N, L+1}\left(\omega_{j}\right)+F\left(\overline{\bar{g}}_{N, L}\left(\omega_{j}\right)\right) G\left(\bar{\Lambda}_{\Psi, Q}\left(\overline{\bar{g}}\left(\cdot, \cdot, \omega_{j}\right)\right)\right)+\overline{\bar{g}}_{N, L+1}\left(\omega_{j}\right)}{2}
$$

as a better estimate. All the numerical results presented below use antithetic variates. In general, the use of control variates, see [5], can be also combined with other variance reduction methods. For example, the control variates technique is based on the knowledge of an estimator $Y_{\star}$, positively correlated with $Y$, whose expected value $\mathbb{E}\left[Y_{\star}\right]$ is known and relatively close to the desired $\mathbb{E}[Y]$, yielding $Y-Y_{\star}+\mathbb{E}\left[Y_{\star}\right]$ as an improved estimator. The estimates presented in this work do not preclude the use of control variates, and even though it is not applied here, it can be a valuable tool in practical computations.

5.2. Numerical results. Now let us introduce some notation to be used later in the description of our numerical results. $\mathrm{E}_{\text {tau }}$ denotes the sample average approximating the $\tau$-discretization error (4.7) and $\mathrm{E}_{\text {tim }}$ denotes the sample average approximation to the $t$-discretization error (4.8). Beside this, denote by $\mathrm{E}_{S}$ the approximation (5.1) to the statistical error $E_{S}$ introduced in (2.14) and by $\mathrm{E}_{\mathrm{tau}, S}$ the approximation (5.1) to the statistical error in the estimation of the $\tau$-discretization error (4.7) by sample averages. Similarly, $\mathrm{E}_{\mathrm{tim}, S}$ denotes the corresponding approximation to the statistical error in the estimation of the expected values in $t$-discretization error (4.8).

5.2.1. Ho-Lee model. The Ho-Lee model has $\xi(x)=\sigma$ and $\lambda_{0}(x)=1$ so $\widetilde{\lambda}_{0}(x)=x$ and (1.2)-(1.3) takes the form

$$
\begin{aligned}
d f(t, \tau) & =\sigma^{2}(\tau-t) d t+\sigma d W(t), \quad 0 \leq t \leq \tau, \\
f(0, \tau) & =f_{0}(\tau)
\end{aligned}
$$


for $\tau \in\left[0, \tau_{\text {max }}\right]$. In this example the initial condition is $f_{0}(\tau)=r_{0}-\frac{\sigma^{2}}{2} \tau^{2}+\int_{0}^{\tau} \vartheta(s) d s$, where $r_{0}$ and $\sigma$ are real positive constants and $\vartheta: \mathbb{R}^{+} \rightarrow \mathbb{R}$ is a given function. Then, the exact solution of (5.2) is

$$
f(t, \tau)=r_{0}-\frac{\sigma^{2}}{2}(\tau-t)^{2}+\int_{0}^{\tau} \vartheta(s) d s+\sigma W(t), \quad 0 \leq t \leq \tau,
$$

which follows the normal distribution and therefore, yields bond prices which are log-normal distributed, allowing the use of Black and Scholes formulas for the pricing of call and put options on bonds.

Setting $\tau_{a}=t_{\max }, F(x)=1-x, G(x)=x, \Psi(x)=x$ and $U(x)=0$ in (1.5)-(1.6), the functional to be computed has the form

$$
\mathbb{E}[\mathcal{F}(f)]=\mathbb{E}\left[\left(1-\int_{0}^{t_{\max }} f(s, s) d s\right)\left(\int_{t_{\max }}^{\tau_{\max }} f\left(t_{\max }, \tau\right) d \tau\right)\right] .
$$

In the numerical experiments we choose $r_{0}=0.05, \sigma=0.01, \vartheta(s)=\frac{1}{10} e^{-s}$. Then $\mathbb{E}[\mathcal{F}(f)]$ is a known function of $t_{\max }$ and $\tau_{\max }$. The first experiment sets $t_{\max }=1.0$ and $\tau_{\max }=2.0$, comparing the efficiency of the (EFD) and (EFE) methods. Table 5.2.1 shows the computational error for both methods and compares the a posteriori approximation of the error with the true computational error for the (EFD) method. Here, a confidence interval for the ratio between the error approximation and the exact computational error, $\mathcal{E}_{c}$, introduced in (2.12), is $[A-B, A+B]$, with $A \equiv \frac{\mathrm{E}_{\mathrm{tim}}+\mathrm{E}_{\mathrm{tau}}}{\left|\mathcal{E}_{c}\right|}$ and $B \equiv \frac{\mathrm{E}_{S}+\mathrm{E}_{\mathrm{tim}, S}+\mathrm{E}_{\mathrm{tau}, S}}{\left|\mathcal{E}_{c}\right|}$. Whenever we use the (EFD) method we call $\mathcal{E}_{C, E F D} \equiv \mathcal{E}_{c}$ and if we use the (EFE) method we call $\mathcal{E}_{C, E F E} \equiv \mathcal{E}_{c}$. Observe that the ratio $A \pm B$ of the a posteriori approximation of the error over the computational error becomes closer and closer to one as we refine the time and maturity partitions, provided that the statistical error is small compared to the $t$-discretization error and the $\tau$-discretization error. In this example, the $t$-discretization gives the largest contribution to the computational error, and there is no practical advantage in the use of the (EFE) method.

\begin{tabular}{|c|c|c|c|}
\hline iseed $=-1$ & $(\mathrm{EFE})$ & \multicolumn{2}{|c|}{$(\mathrm{EFD})$} \\
\hline$N=L$ & $\mathcal{E}_{C, \text { EFE }}$ & $\mathcal{E}_{C, \text { EFD }}$ & {$[A-B, A+B]$} \\
\hline 5 & $-8.40 \times 10^{-4}$ & $-8.25 \times 10^{-4}$ & {$[0.97,0.97]$} \\
\hline 10 & $-4.16 \times 10^{-4}$ & $-4.08 \times 10^{-4}$ & {$[0.98,0.99]$} \\
\hline 20 & $-2.07 \times 10^{-4}$ & $-2.03 \times 10^{-4}$ & {$[0.98,1.00]$} \\
\hline
\end{tabular}

Table 5.2.1 Comparing the (EFD) and (EFE) methods in the Ho-Lee model approximating functional (5.3) with $M=5000$ and $c_{0}=1.65$.

5.2.2. Vasicek model. The Vasicek model has $\xi(x)=\sigma$ and $\lambda_{0}(x)=e^{-\alpha x}$, so

$$
\widetilde{\lambda}_{0}(x)=\frac{1}{\alpha} e^{-\alpha x}\left(1-e^{-\alpha x}\right)
$$

and the forward rate equation $1.2(1.3)$ becomes

$$
\begin{aligned}
d f(t, \tau) & =\frac{\sigma^{2}}{\alpha}\left(1-e^{-\alpha(\tau-t)}\right) e^{-\alpha(\tau-t)} d t+\sigma e^{-\alpha(\tau-t)} d W(t), \quad 0 \leq t \leq \tau, \\
f(0, \tau) & =f_{0}(\tau)
\end{aligned}
$$

for $\tau \in\left[0, \tau_{\max }\right]$. In this example the initial condition is

$$
f_{0}(\tau)=\left(r_{0}-\frac{\vartheta}{\alpha}\right) e^{-\alpha \tau}+\frac{\vartheta}{\alpha}-\frac{\sigma^{2}}{2 \alpha^{2}}\left(1-e^{-\alpha \tau}\right)^{2}, \quad \tau \in\left[t, \tau_{\max }\right],
$$

where $r_{0}, \sigma, \alpha$ and $\vartheta$ are given positive constants. The solution of (5.4) is then

$$
\begin{aligned}
f(t, \tau)= & e^{-\alpha(\tau-t)}\left[e^{-\alpha t}\left(r_{0}-\frac{\vartheta}{\alpha}\right)+\sigma \int_{0}^{t} e^{-\alpha(t-s)} d W(s)\right] \\
& +\frac{\vartheta}{\alpha}-\frac{\sigma^{2}}{2 \alpha^{2}}\left(1-e^{-\alpha(\tau-t)}\right)^{2}, \quad 0 \leq t \leq \tau,
\end{aligned}
$$

which is normally distributed and yields bond prices that are lognormal, as in the Ho-Lee model.

Here we set $\tau_{a}=t_{\max }=0.3, \tau_{\max }=6.0$, and approximate again the functional defined in (5.3). In addition, we take $r_{0}=0.03, \alpha=1.0, \sigma=0.01$ and $\vartheta=0.05$. Table 5.2 .2 displays the computational 
errors for the (EFD) and (EFE) methods and compares the a posteriori approximation of the error with the true error for the (EFD) method. Observe that the ratio $A \pm B$ of the a posteriori approximation of the error over the computational error becomes closer and closer to 1 as we refine the time and maturity partitions, provided that the statistical error is small compared to the $t$ - and $\tau$-discretization error.

\begin{tabular}{|c|c|c|c|}
\hline iseed $=-1$ & $(\mathrm{EFE})$ & \multicolumn{2}{|c|}{$(\mathrm{EFD})$} \\
\hline$N=L$ & $\mathcal{E}_{C, \text { EFE }}$ & $\mathcal{E}_{C, \text { EFD }}$ & {$[A-B, A+B]$} \\
\hline 5 & $-2.30 \times 10^{-5}$ & $-2.07 \times 10^{-5}$ & {$[1.92,1.95]$} \\
\hline 10 & $-2.05 \times 10^{-5}$ & $-1.95 \times 10^{-5}$ & {$[1.03,1.05]$} \\
\hline 20 & $-1.06 \times 10^{-5}$ & $-1.00 \times 10^{-5}$ & {$[0.99,1.02]$} \\
\hline
\end{tabular}

Table 5.2.2. Comparing the (EFD) and (EFE) methods in the Vasicek model approximating functional (5.3) with $M=5000$ and $c_{0}=1.65$.

5.2.3. The Cox-Ingersoll-Ross (CIR) model. Consider the following (CIR) short rate model

$$
r(t)=r_{0}+\int_{0}^{t}(\vartheta-\alpha r(s)) d s+\int_{0}^{t} \sigma \sqrt{r(s)} d W(s), \quad t \geq 0,
$$

where $\vartheta, \alpha$ and $\sigma$ are real constants. To connect the solution $r(t)$ of 5.5 to the diagonal value $f(t, t)$ of the solution of an HJM problem, consider, first, the solution $B=B(t ; \tau)$ of the following Riccati differential equation (see [1]):

$$
\begin{aligned}
\partial_{t} B(t ; \tau) & =\frac{1}{2} \sigma^{2} B^{2}(t ; \tau)+\alpha B(t ; \tau)-1, \quad t \in[0, \tau], \quad \tau \geq 0, \\
B(\tau ; \tau) & =0,
\end{aligned}
$$

which has the form $B(t ; \tau)=\psi(\tau-t)$ where

$$
\psi(x)=-\frac{\alpha}{\sigma^{2}}+\frac{2}{\sigma^{2}} \quad \widetilde{\gamma}_{0} \frac{\sinh \left(\widetilde{\gamma}_{0} x\right)+\frac{\alpha}{2 \widetilde{\gamma}_{0}} \cosh \left(\widetilde{\gamma}_{0} x\right)}{\cosh \left(\widetilde{\gamma}_{0} x\right)+\frac{\alpha}{2 \widetilde{\gamma}_{0}} \sinh \left(\widetilde{\gamma}_{0} x\right)} \quad \text { and } \quad \widetilde{\gamma}_{0}:=\frac{1}{2} \sqrt{2 \sigma^{2}+\alpha^{2}} .
$$

Provided $\xi(x)=\sigma \sqrt{\max \{x, 0\}}$ and $\lambda_{0}(x)=\psi^{\prime}(x)$, then $\widetilde{\lambda}_{0}(x)=\psi^{\prime}(x) \psi(x)$ and the stochastic function

$$
f(t, \tau)=r(t) \psi^{\prime}(\tau-t)+\vartheta \psi(\tau-t)
$$

solves (1.2)-(1.3) with the initial condition $f_{0}(\tau)=r_{0} \psi^{\prime}(\tau)+\vartheta \psi(\tau)$. Taking into account that $\psi^{\prime}(0)=1$ and $\psi(0)=0$, it follows that $f(t, t)=r(t)$.

Setting $\tau_{a}=t_{\max }, F(x)=e^{-x}, G(x)=\max \left\{e^{-x}-K_{0}, 0\right\}, \Psi(x)=x$ and $U(x)=0$ in (1.5][1.6), the functional to compute in this example takes the form

$$
\mathbb{E}[\mathcal{F}(f)]=\mathbb{E}\left[\exp \left(-\int_{0}^{t_{\max }} f(s, s) d s\right) \max \left\{\exp \left(-\int_{t_{\max }}^{\tau_{\max }} f\left(t_{\max }, \tau\right) d \tau\right)-K_{0}, 0\right\}\right] .
$$

In the numerical experiments we choose $r_{0}=0.15, \alpha=1.0, \sigma=0.1, \vartheta=0.05, t_{\max }=5.0, \tau_{\max }=8.0$ and $K_{0}=0.5$. Table 5.2 .3 shows the computational errors for the (EFD) and (EFE) methods and the ratio between the approximation of the computational error and the exact computational error for (EFD) method. There is no practical difference in this case between the (EFD) and the (EFE) method since the computational error is mainly $t$-discretization error and the $\tau$-discretization error is relatively unimportant.

In order to have smooth coefficients in the HJM model (1.2]1.3) we approximate the function $\sqrt{\max \{x, 0\}}$ in the diffusion term by a Lipschitz function globally defined in $\mathbb{R}$ (cf. 9] p. 252),

$$
\sqrt{\max \{x, 0\}} \approx \sqrt{\frac{1}{2}\left(x+\sqrt{x^{2}+\delta}\right)}
$$

where $\delta$ is a small positive constant. Observe that after this regularization the value of the functional $\mathbb{E}[\mathcal{F}(f)]$ depends on $\delta$. In the computations $\delta$ has been taken small enough to make this dependence negligible with respect to the size of the computational error. In this example we compute an accurate 


\begin{tabular}{|c|c|c|c|}
\hline iseed $=-1$ & $(\mathrm{EFE})$ & \multicolumn{2}{|c|}{$(\mathrm{EFD})$} \\
\hline$N=L$ & $\mathcal{E}_{C, E F E}$ & $\mathcal{E}_{C, E F D}$ & {$[A-B, A+B]$} \\
\hline 5 & $1.23 \times 10^{-2}$ & $1.21 \times 10^{-2}$ & {$[0.31,0.44]$} \\
\hline 10 & $5.83 \times 10^{-3}$ & $5.39 \times 10^{-3}$ & {$[0.91,0.95]$} \\
\hline 20 & $2.76 \times 10^{-3}$ & $2.79 \times 10^{-3}$ & {$[0.89,0.94]$} \\
\hline
\end{tabular}

Table 5.2.3. Comparing the (EFD) and (EFE) methods in the (CIR) model approximating functional (5.6) with $M=2000$ and $c_{0}=1.65$.

numerical approximation of the exact $\mathbb{E}[\mathcal{F}(f)]$ from (5.6), via the Feynman-Kac representation formula, using a numerical solution of the following backward PDE (cf. [20] p. 313),

$$
v_{t}+(\vartheta-\alpha r) v_{r}+\frac{1}{2} \sigma^{2} r v_{r r}-r v=0, \quad t \in\left[0, t_{\max }\right], \quad r \in\left[0, r_{\max }\right],
$$

with final datum $v\left(t_{\max }, r\right)=\left(B\left(r, t_{\max }, \tau_{\max }\right)-K_{0}\right)^{+}$, where $B\left(r, t_{\max }, \tau_{\max }\right)$ denotes the $(\mathrm{CIR})$ value for a bond with contracting time $t_{\max }$, maturity time $\tau_{\max }$ and short rate at $t_{\max }$ equal to $r$. We also use the boundary conditions

$$
v_{t}(t, 0)+\alpha v_{r}(t, 0)=0, \quad v\left(t, r_{\max }\right)=0,
$$

for $t \in\left[0, t_{\max }\right]$. The value of $r_{\max } \gg \frac{\vartheta}{\alpha}$ is taken sufficiently large so that the homogeneous Dirichlet boundary at $r=r_{\max }$ has a negligible effect on the numerical approximation for $v(0,0.15)=\mathbb{E}[\mathcal{F}(f)]$. The spatial discretization is a centered finite differences scheme and the time stepping is done by a diagonally implicit Runge Kutta method, namely the DIRK2 method, see 8 . Another way to estimate the exact solution with high accuracy is to use a formula based on the $\chi^{2}$ distribution (see [19], pp. 187-193 for details).

5.2.4. A two-factor Gaussian model. A two-factor model has randomness introduced by two scalar independent Wiener processes $W_{1}, W_{2}$. In particular, for a two-factor Gaussian model we have $\xi(x)=1$, $\lambda_{0,1}(x)=\sigma_{1}$ and $\lambda_{0,2}(x)=\sigma_{2} e^{-\frac{a_{2} x}{2}}$, where $\sigma_{1}, \sigma_{2}$ and $a_{2}$ are real positive constants. Thus (1.2)-(1.3) takes the form

$$
\begin{aligned}
d f(t, \tau)= & {\left[\begin{array}{ll}
\left(\sigma_{1}\right)^{2} & \left.(\tau-t)+\frac{2\left(\sigma_{2}\right)^{2} e^{-\frac{a_{2}(\tau-t)}{2}}}{a_{2}}\left(1-e^{-\frac{a_{2}(\tau-t)}{2}}\right)\right] d t \\
& +\sigma_{1} d W_{1}(t)+\sigma_{2} e^{-\frac{a_{2}(\tau-t)}{2}} d W_{2}(t), \quad 0 \leq t \leq \tau,
\end{array}\right.} \\
f(0, \tau)= & f_{0}(\tau)
\end{aligned}
$$

for $\tau \in\left[0, \tau_{\max }\right]$. Here the initial condition is $f_{0}(\tau)=b_{0}+b_{1} e^{-k \tau}$ where $b_{0}, b_{1}$ and $k$ are real constants. Then, the exact solution of (5.7) is normal distributed as in the Ho-Lee and Vasicek models, so explicit formulas are available for the pricing of put and call options with bonds as underlyings.

In the numerical experiment we take $\sigma_{1}=0.02, \sigma_{2}=0.01, a_{2}=0.5$, and compute with the functional defined in (5.6) with strike $K_{0}=0.5, t_{\max }=1$ and $\tau_{\max }=3$. For the initial condition we set $b_{0}=0.0759$, $b_{1}=-0.0439$ and $k=0.4454$. Table 5.2.4 shows the computational errors for the (EFD) and (EFE) methods and the ratio between the approximation of the computational error and the exact computational error for method (EFD).

\begin{tabular}{|c|c|c|c|}
\hline iseed $=-1$ & $(\mathrm{EFE})$ & \multicolumn{2}{|c|}{ (EFD) } \\
\hline$N=L$ & $\mathcal{E}_{C, E F E}$ & $\mathcal{E}_{C, E F D}$ & {$[A-B, A+B]$} \\
\hline 5 & $-5.15 \times 10^{-4}$ & $-6.90 \times 10^{-4}$ & {$[0.98,1.02]$} \\
\hline 10 & $-2.78 \times 10^{-4}$ & $-3.50 \times 10^{-4}$ & {$[0.96,1.05]$} \\
\hline
\end{tabular}

Table 5.2.4. Comparing the (EFD) and (EFE) methods in the two-factor Gaussian model approximating functional (5.6) with $M=40000$ and $c_{0}=1.65$. 


\section{ACKNOWLEDGEMENTS}

This work has been partially supported by: The Swedish National Network in Applied Mathematics (NTM) 'Numerical approximation of stochastic differential equations' (NADA, KTH), The EU-TMR project HCL \# ERBFMRXCT960033, UdelaR and UdeM in Uruguay, The Swedish Research Council for Engineering Science (TFR) Grant\#222-148, The VR project 'Effektiva numeriska metoder för stokastiska differentialekvationer med tillämpningar' (NADA, KTH), the European Union's Seventh Framework Programme (FP7-REGPOT-2009-1) under grant agreement no. 245749 'Archimedes Center for Modeling, Analysis and Computation' (University of Crete, Greece), The University of Crete, and The King Abdullah University of Science and Technology (KAUST).

\section{REFERENCES}

[1] M. Baxter and A. Rennie. Financial Calculus: An introduction to derivate pricing. Cambridge University Press, 1996.

[2] Å. Björck and G. Dahlquist. Numerical methods. Prentice-Hall Inc., Englewood Cliffs, N.J., 1974. Translated from the Swedish by Ned Anderson, Prentice-Hall Series in Automatic Computation.

[3] T. Björk. Arbitrage theory in continuous time. Oxford University Press Inc., 1998.

[4] P. Boyle, M. Broadie, and P. Glasserman. Monte Carlo methods for security pricing. In Option pricing, interest rates and risk management, pp. 185-238. Cambridge Univ. Press, Cambridge, 2001.

[5] R. E. Caflisch. Monte Carlo and quasi-Monte Carlo methods. In Acta numerica, 1998, pp. 1-49. Cambridge Univ. Press, Cambridge, 1998.

[6] A. Carverhill. A note on the models of Hull and White for pricing options on the term structure. Journal of Fixed Income, 5 (1995), no 2, pp. 89-96.

[7] A. Carverhill and K. Pang. Efficient and flexible bond option valuation in the Heath, Jarrow and Morton framework. Journal of Fixed Income, 5 (1995), no 2, pp. 70-77.

[8] K. Dekker and J. G. Verwer. Stability of Runge-Kutta methods for stiff nonlinear differential equations. North-Holland Publishing Co., Amsterdam, 1984.

[9] D. Duffie. Dynamic asset pricing theory. Princeton University Press, Princeton, New Jersey, second edition, 1996.

[10] R. Durett. Probability: theory and examples. Duxbury Press, 1994.

[11] G. S. Fishman. Monte Carlo. Springer-Verlag, New York, 1996. Concepts, algorithms, and applications.

[12] J. M. Hammersley and K. W. Morton. A new Monte Carlo technique: antithetic variates. Proc. Cambridge Philos. Soc., 52 (1956), pp. 449-475.

[13] D. Heath, R. Jarrow, and A. Morton. Bond pricing and the term structure of interest rates: a discrete time approximation. Journal of Financial and Quantitative Analysis, 25 (1990), pp. 419-440.

[14] D. Heath, R. Jarrow, and A. Morton. Bond pricing and the term structure of interest rates: a new methodology for contingent claims valuation. Econometrica, 60 (1992), pp. 77-105.

[15] J. Hull. Options, Futures and other Derivatives. Prentice Hall, Upper Saddle River, NJ, 1993.

[16] N. Metropolis and S. Ulam. The Monte Carlo method. J. Amer. Statist. Assoc., 44 (1949), pp. 335-341.

[17] K.-S. Moon, A. Szepessy, R. Tempone, and G.E. Zouraris. Convergence rates for adaptive weak approximation of stochastic differential equations. Stochastic Analysis and Applications, 23 (2005), pp. 511-558.

[18] W.H. Press. Numerical recipes in FORTRAN: the art of scientific computing. Cambridge University Press, Cambridge, United Kingdom, second edition, 1992.

[19] R. Rebonato. Interest-Rate Option Models. John Wiley \& Sons Ltd, 1996.

[20] S. Shreve. Stochastic calculus and finance. Lecture Notes, 1996 (http://www.stat.berkeley.edu/users/evans/shreve.pdf).

[21] A. Szepessy, R. Tempone, and G. E. Zouraris. Adaptive weak approximation of stochastic differential equations. Comm. Pure Appl. Math., 54 (2001), pp. 1169-1214.

[22] R. Tempone. Numerical Complexity Analysis of Weak Approximation of Stochastic Differential Equations. Ph.D Dissertation, KTH, Stockholm, Sweden, 2002. 